\title{
The motive of a classifying space
}

\author{
Burt Totaro
}

The Chow group of algebraic cycles generally does not satisfy the Künneth formula. Nonetheless, there are some schemes $X$ over a field $k$ which satisfy the Chow Künneth property that the product $C H_{*} X \otimes_{\mathbf{z}} C H_{*} Y \rightarrow C H_{*}\left(X \times_{k} Y\right)$ is an isomorphism for all separated schemes $Y$ of finite type over $k$. The Chow Künneth property implies the weak Chow Künneth property that $C H_{*} X \rightarrow C H_{*}\left(X_{F}\right)$ is surjective for every finitely generated field $F$ over $k$ (or, equivalently, for every extension field $F$ of $k$ ). We characterize several properties of this type. (We also prove versions of all our results with coefficients in a given commutative ring.)

Our characterizations of Künneth properties are: first, a smooth proper scheme $X$ over $k$ satisfies the weak Chow Künneth property if and only if the Chow motive of $X$ is a summand of a direct sum of Tate motives (Theorem 4.1). (This is related to known results by Bloch, Srinivas, Jannsen, Kimura, and others.) A more novel result is about an arbitrary separated scheme $X$ of finite type over $k$. We say that $X$ satisfies the motivic Künneth property if the Künneth spectral sequence converges to the motivic homology groups of $X \times_{k} Y$ for all $Y$. (Motivic homology groups are also called higher Chow groups; they include the usual Chow groups as a special case.) We show that a $k$-scheme $X$ satisfies the motivic Künneth property if and only if the motive of $X$ in Voevodsky's derived category of motives is a mixed Tate motive (Theorem 7.2). (An example of a scheme with these properties is any linear scheme, as discussed in section 5.) Finally, if a smooth but not necessarily proper $k$-variety $X$ satisfies the weak Chow Künneth property, then the birational motive of $X$ in the sense of Rost and Kahn-Sujatha is isomorphic to the birational motive of a point (Corollary 2.2.).

The last result cannot be strengthened to say that the motive of $X$ is mixed Tate; one has to consider motivic homology groups to get that conclusion. For example, the complement $X$ of a curve of genus 1 in the affine plane has the Chow Künneth property, since $C H_{i+2}\left(X \times_{k} Y\right) \cong C H_{i} Y$ for all separated $k$-schemes $Y$ of finite type and all $i$; but the motive of $X$ is not mixed Tate.

As an application of these general results, we disprove the weak Chow Künneth property for some classifying spaces $B G$. For an affine group scheme $G$ of finite type over a field $k$, Morel-Voevodsky and I constructed $B G$ as a direct limit of smooth $k$-varieties, quotients by $G$ of open subsets of representations of $G$ over $k$ [39, section 4.2], [53, 55]. As a result, the Chow ring of $B G$ makes sense. The Chow ring of $B G$ tensored with the rationals is easy to compute; for example, if $G$ is finite, then $C H^{i}(B G) \otimes \mathbf{Q}=0$ for $i>0$. The challenge is to understand the integral or $\bmod p$ Chow ring of $B G$.

For many finite groups $G$ and fields $k$, the classifying space $B G$ over $k$ satisfies the Chow Künneth property that $C H^{*} B G \otimes_{\mathbf{z}} C H_{*} Y \cong C H_{*}\left(B G \times_{k} Y\right)$ for all separated $k$-schemes $Y$ of finite type. For example, an abelian $p$-group $G$ of exponent 
$e$ has the Chow Künneth property when $k$ is a field of characteristic not $p$ that contains the eth roots of unity. The Chow Künneth property also holds for many other groups, such as wreath products of abelian groups [55, Lemma 2.12]. As a result, [55, Chapter 17] asked whether every finite group $G$ has the Chow Künneth property over a field $k$ which contains enough roots of unity. This would imply the weak Chow Künneth property that $C H^{*} B G_{k} \rightarrow C H^{*} B G_{F}$ is surjective for every extension field $F$ of $k$.

In this paper, we give the first examples of finite groups for which the Chow Künneth property fails. For any finite group $G$ such that $B G$ has nontrivial unramified cohomology, there is a finitely generated field $F$ over $\overline{\mathbf{Q}}$ such that $C H^{*} B G_{\overline{\mathbf{Q}}} \rightarrow C H^{*} B G_{F}$ is not surjective (Corollary 3.1). We also find a field $E$ containing $\overline{\mathbf{Q}}$ such that $C H^{i}\left(B G_{E}\right) / p$ is infinite for some $i$ and some prime number $p$ (Corollary 3.2); this answers another question in [55, Chapter 18]. In particular, the ring $C H^{*}\left(B G_{E}\right) / p$ is not noetherian in such an example.

As recalled in section 2, there are groups of order $p^{5}$ for any odd prime $p$, and groups of order $2^{6}$, which have nontrivial unramified cohomology. This is surprisingly sharp. In fact, the Chow ring $C H^{*} B G_{k}$ of a $p$-group $G$ is independent of the field $k$ containing $\overline{\mathbf{Q}}$, and consists of transferred Euler classes of representations, when $G$ is a $p$-group of order at most $p^{4}$ [55, Theorem 11.1, Theorem 17.4]. Moreover, the weak Chow Künneth property holds for all groups of order $2^{5}$ (Theorem 10.1).

Finally, section 8 defines the compactly supported motive, in Voevodsky's derived category of motives, for a quotient stack over a field. In particular, we get a notion of the compactly supported motive $M^{c}(B G)$ for an affine group scheme $G$. Once we have this definition, we can ask when $M^{c}(B G)$ is a mixed Tate motive. This property is equivalent to the motivic Künneth property for $B G$, and so it implies the Chow Künneth property for $B G$. In particular, $B G$ is not mixed Tate for the groups of order $p^{5}$ discussed above. On the other hand, we show that many familiar finite groups, such as the finite general linear groups in cross-characteristic and the symmetric groups, are mixed Tate (Theorems 9.11 and 9.12).

The introduction to section 9 discusses six properties of finite groups. It would be interesting to find out whether all six properties are equivalent, as the known examples suggest. The properties are: stable rationality of $B G$ (say, over the complex numbers), meaning stable rationality of quotient varieties $V / G$; triviality for the birational motive of $B G$ (or equivalently, of quotient varieties $V / G$ ); Ekedahl's class of $B G$ in the Grothendieck ring of varieties being equal to 1 [20]; the weak Chow Künneth property of $B G$; the Chow Künneth property of $B G$; and the mixed Tate property of $B G$.

I thank Christian Haesemeyer, Tudor Padurariu, and a referee for their suggestions. This work was supported by NSF grant DMS-1303105.

\section{Contents}

1 Notation 3

2 Birational motives 3 
3 Failure of the weak Chow Künneth property for finite groups

4 The weak Chow Künneth property for smooth proper $k$-schemes 10

5 The triangulated category of motives 13

6 A Künneth spectral sequence for motivic homology 18

7 The motivic Künneth property 19

8 The motive of a quotient stack 23

9 The mixed Tate property for classifying spaces 32

10 Groups of order 32

\section{Notation}

A variety over a field $k$ means an integral separated scheme of finite type over $k$. A variety $X$ over $k$ is geometrically integral if $X_{\bar{k}}:=X \times_{\operatorname{Spec}(k)} \operatorname{Spec}(\bar{k})$ is an integral scheme (where $\bar{k}$ is an algebraic closure of $k$ ), or equivalently if $X_{E}$ is integral for every extension field $E$ of $k$ [24, Definition IV.4.6.2].

Let $X$ be a scheme of finite type over a field $k$. The Chow group $C H_{i} X$ is the group of $i$-dimensional algebraic cycles on $X$ modulo rational equivalence. A good reference is Fulton [22, Chapter 1]. We write $C H_{i}(X ; R)=C H_{i}(X) \otimes_{\mathbf{z}} R$ for a commutative ring $R$.

For a smooth scheme $X$ over $k$, understood to be of finite type over $k$, we write $C H^{i} X$ for the Chow group of codimension- $i$ cycles on $X$. For $X$ smooth over $k$, the groups $C H^{*} X$ have a ring structure given by intersecting cycles [22, Chapter 6].

\section{Birational motives}

In this section, we give several equivalent characterizations of those smooth proper varieties $X$ whose birational motive in the sense of Rost [32, Appendix RC] and Kahn-Sujatha [31, equation (2.5)] is isomorphic to the birational motive of a point. The statement includes Merkurjev's theorem that the Chow group of 0-cycles on $X$ is unchanged under field extensions if and only if the unramified cohomology of $X$ in the most general sense is trivial [38. It seems to be new that these properties are also equivalent to all the Chow groups of $X$ being supported on a divisor. Note that these properties are not equivalent to $\mathrm{CH}_{0}$ being supported on a divisor; for example, the product of $\mathbf{P}^{1}$ with a curve $C$ of genus at least 1 has $C H_{0}$ supported on a divisor, while $\mathrm{CH}_{1}$ is not supported on a divisor. Also, unlike many earlier results in this area, we work with an arbitrary coefficient ring, not just the rational numbers.

We will use the equivalence of Theorem 2.1 to give the first counterexamples to the Chow Künneth property for the classifying space of a finite group over an algebraically closed field (section 3 ). 
Theorem 2.1. Let $X$ be a smooth proper variety over a field $k$, and let $R$ be a nonzero commutative ring. The following are equivalent.

(1) For every finitely generated field $\mathrm{F} / k, \mathrm{CH}_{0}(\mathrm{X} ; \mathrm{R}) \rightarrow \mathrm{CH}_{0}\left(\mathrm{X}_{F} ; R\right)$ is surjective.

(2) For every field $F / k, C H_{0}(X ; R) \rightarrow C H_{0}\left(X_{F} ; R\right)$ is an isomorphism, and both groups map isomorphically to $R$ by the degree.

(3) The birational motive of $X$ (in the sense of Kahn-Sujatha) with $R$ coefficients is isomorphic to the birational motive of a point.

(4) For every $R$-linear cycle module $M$ over $k$ (in the sense of Rost [46]), the homomorphism $M(k) \rightarrow M(k(X))_{n r}$ is an isomorphism. (That is, $X$ has trivial unramified cohomology in the most general sense.)

(5) There is a closed subset $S \subsetneq X$ such that

$$
C H_{i}(X ; R) / C H_{i}(S ; R) \rightarrow C H_{i}\left(X_{F} ; R\right) / C H_{i}\left(S_{F} ; R\right)
$$

is surjective for all finitely generated fields $F / k$ and all integers $i$. (That is, all the Chow groups of $X$ are constant outside a divisor.)

(6) The variety $X$ is geometrically integral, and there is a closed subset $S \subsetneq X$ such that $C H_{i}\left(X_{F} ; R\right) / C H_{i}\left(S_{F} ; R\right)=0$ for all fields $F / k$ and all $i<\operatorname{dim}(X)$.

For the coefficient ring $R=\mathbf{Q}$ which has been considered most often, there are other equivalent statements: instead of considering all finitely generated extension fields of $k$ in (1) or (5), one could consider a single algebraically closed field of infinite transcendence degree over $k$. This gives equivalent conditions, because $C H_{*}\left(X_{F} ; \mathbf{Q}\right) \rightarrow C H_{*}\left(X_{E} ; \mathbf{Q}\right)$ is injective for every scheme $X$ over $F$ and every inclusion of fields $F \hookrightarrow E$. On the other hand, for the coefficient $\operatorname{ring} R=\mathbf{F}_{p}$ which is of most interest for the classifying space of a finite group, it would not be enough to consider algebraically closed extension fields in Theorem 2.1. This follows from Suslin's rigidity theorem: for every extension of algebraically closed fields $F \hookrightarrow E$, every $k$-scheme $X$ over $F$, and every prime number $p$ invertible in $F, C H_{*}\left(X_{F} ; \mathbf{F}_{p}\right)$ maps isomorphically to $C H_{*}\left(X_{E} ; \mathbf{F}_{p}\right)$ [51, Corollary 2.3.3].

Corollary 2.2. Let $k$ be a perfect field which admits resolution of singularities (for example, any field of characteristic zero). Let $U$ be a smooth variety over $k$, not necessarily proper. Let $R$ be a commutative ring. If $C H^{*}(U ; R) \rightarrow C H^{*}\left(U_{F} ; R\right)$ is surjective for every finitely generated field $F$ over $k$, then the birational motive of $U$ with coefficients in $R$ is isomorphic to the birational motive of a point.

Proof. (Corollary 2.2) By resolution of singularities, there is a regular compactification $X$ of $U$ over $k$, with $U=X-S$ for some closed subset $S$. Since $k$ is perfect, the regular scheme $X$ is smooth over $k$. Let us index Chow groups by dimension. We use the basic exact sequence for Chow groups [22, Proposition 1.8]:

Lemma 2.3. Let $X$ be a scheme of finite type over a field $k$. Let $Z$ be a closed subscheme. Then the proper pushforward and flat pullback maps fit into an exact sequence

$$
C H_{i}(Z) \rightarrow C H_{i}(X) \rightarrow C H_{i}(X-Z) \rightarrow 0 .
$$

In the case at hand, it follows that $C H_{*}(U ; R)$ is isomorphic to $C H_{*}(X ; R) / C H_{*}(S ; R)$. So the assumption on $U$ implies condition (5) in Theorem 2.1. The birational motive of $U$ is (by definition) the same as that of $X$. By Theorem 2.1, the birational motive of $X$ with coefficients in $R$ is isomorphic to the birational motive of a point. 
Proof. (Theorem 2.1) Assume condition (1). That is, $C H_{0}(X ; R) \rightarrow C H_{0}\left(X_{F} ; R\right)$ is surjective for every finitely generated field $F / k$. Let $n$ be the dimension of $X$. The generic fiber of the diagonal $\Delta$ in $C H_{n}\left(X \times_{k} X\right)$ via projection to the first copy of $X$ is a zero-cycle in $C H_{0} X_{k(X)}$. By our assumption, the class $[\Delta]$ in $C H_{0}\left(X_{k(X)} ; R\right)$ is the image of some zero-cycle $\alpha \in C H_{0}(X ; R)$. For a variety $Y$ over $k$, the Chow groups of $X_{k(Y)}$ are the direct limit of the Chow groups of $X \times_{k} U$, where $U$ runs over all nonempty open subsets of $Y$. (Note that an $i$-dimensional cycle on $X \times{ }_{k} U$ gives a cycle of dimension $i-\operatorname{dim}(Y)$ on the generic fiber $X_{k(Y)}$.) Therefore, we can write

$$
\Delta=X \times \alpha+B
$$

in $C H_{n}\left(X \times_{k} X ; R\right)$, where $B$ is a cycle supported on $S \times X$ for some closed subset $S \subsetneq X$. Here we are using the basic exact sequence for Chow groups (Lemma 2.3).

As a correspondence, the diagonal $\Delta$ induces the identity map from $C H_{i}(X ; R)$ to $C H_{i}(X ; R)$ for any $i$. For this purpose, think of $\Delta$ as a correspondence from the first copy of $X$ to the second. It follows that for any extension field $F$ of $k$ and any zero-cycle $\beta$ in $C H_{0}\left(X_{F} ; R\right), \beta=\Delta_{*}(\beta)=(X \times \alpha)_{*}(\beta)=\operatorname{deg}(\beta) \alpha$. Thus the $R$-module $C H_{0}\left(X_{F} ; R\right)$ is generated by $\alpha$ for every field $F / k$. Moreover, $\alpha$ has degree 1 , and so the degree map $\operatorname{deg}: C H_{0}\left(X_{F} ; R\right) \rightarrow R$ is an isomorphism. We have proved condition (2).

Condition (3) is immediate from (2). Namely, for any smooth proper varieties $X$ and $Y$ over $k$, the set of morphisms from the birational motive of $X$ (with $R$ coefficients) to the birational motive of $Y$ is defined to be $C H_{0}\left(Y_{k(X)} ; R\right)$ 31, equation (2.5)]. So, for a point $p=\operatorname{Spec}(k)$, we have

$$
\begin{aligned}
\operatorname{Hom}_{\text {bir }}(p, p) & =R, \\
\operatorname{Hom}_{\mathrm{bir}}(X, p) & =C H_{0}(\operatorname{Spec}(k(X)) ; R)=R, \\
\operatorname{Hom}_{\mathrm{bir}}(p, X) & =C H_{0}(X ; R), \\
\operatorname{Hom}_{\mathrm{bir}}(X, X) & =C H_{0}\left(X_{k(X)} ; R\right) .
\end{aligned}
$$

By (2), we know that $C H_{0}(X ; R)$ and $C H_{0}\left(X_{k(X)} ; R\right)$ both map isomorphically to $R$ by the degree map; so $X$ has the birational motive of a point. It is now clear that (1), (2), and (3) are equivalent.

When $R=\mathbf{Z}$, Merkurjev proved that (4) is equivalent to (1) and (2) [38, Theorem 2.11]. The proof works with any coefficient ring $R$. For example, to see that (3) implies (4), it suffices to check that an element of $\mathrm{CH}_{0}\left(Y_{k(X)} ; R\right)$ determines a pullback map from unramified cohomology of $Y$ (with coefficients in any $R$-linear cycle module over $k$ ) to unramified cohomology of $X$.

Now we show that (1) (or equivalently, (2), (3), or (4)) implies (5) and (6). Given (1), we have a decomposition of the diagonal as above,

$$
\Delta=X \times \alpha+B
$$

in $C H_{n}\left(X \times_{k} X ; R\right)$, where $B$ is a cycle supported on $S \times X$ for some closed subset $S \subsetneq X$. Now use the correspondence $\Delta$ to pull cycles back from the second copy of $X$ to the first; again, it induces the identity on Chow groups. It follows that for any extension field $F$ of $k$ and any cycle $\beta$ in $C H_{i}\left(X_{F} ; R\right)$ with $i<n$, we have $\beta=$ $\Delta^{*}(\beta)=B^{*}(\beta)$, which is a cycle supported in $S$. Thus $C H_{i}\left(S_{F} ; R\right) \rightarrow C H_{i}\left(X_{F} ; R\right)$ is surjective for all $i<n$. 
To prove (6), we also have to show that $X$ is geometrically integral. Since $X$ is smooth and proper over $k, C H_{n}\left(X_{\bar{k}} ; R\right)$ is the free $R$-module on the set of irreducible components of $X_{\bar{k}}$, and the cycle $[X]$ is the element $(1, \ldots, 1)$ in this module. But for any irreducible component $Y$ of $X_{\bar{k}}$, with class $(1,0, \ldots, 0)$ in $C H_{n}\left(X_{\bar{k}} ; R\right)$, we have $[Y]=\Delta^{*}[Y]=(X \times \alpha)^{*}[Y] \in R \cdot[X]=R \cdot(1, \ldots, 1)$ in $C H_{n}\left(X_{\bar{k}} ; R\right)$. Since the ring $R$ is not zero, it follows that $X_{\bar{k}}$ is irreducible. This proves (6) and hence the weaker statement (5).

Finally, we prove that (5) implies (1), which will complete the proof. This part of the argument seems to be new. We are assuming that there is a closed subset $S \subsetneq X$ such that $C H_{i}(X ; R) / C H_{i}(S ; R) \rightarrow C H_{i}\left(X_{F} ; R\right) / C H_{i}\left(S_{F} ; R\right)$ is surjective for all finitely generated fields $F / k$ and all integers $i$. Taking $i=n$, it follows that $X$ is geometrically integral (using that $R$ is not zero). As above, let $[\Delta]$ denote the generic fiber in $C H_{0}\left(X_{k(X)} ; R\right)$ of the diagonal $\Delta$ in $C H_{n}\left(X \times_{k} X ; R\right)$. We will show by descending induction on $j$ that for each $0 \leq j \leq n$, there is a closed subset $T_{j}$ of $X$ of dimension at most $j$ such that $[\Delta]$ is the image of a zero-cycle $\alpha_{j}$ on $\left(T_{j}\right)_{k(X)}$. This is clear for $j=n$, by taking $T_{n}=X$.

Suppose we have a closed subset $T_{j}$ and a zero-cycle $\alpha_{j}$ as above, for an integer $1 \leq j \leq n$. Then $\alpha_{j}$ is the generic fiber (with respect to the first projection) of some $n$-dimensional cycle $A_{j}$ on $X \times_{k} T_{j}$. Let $T_{j 1}, \ldots, T_{j m}$ be the irreducible components of dimension $j$ in $T_{j}$, and $Z$ the union of any irreducible components of dimension less than $j$ in $T_{j}$. We can write $A_{j}$ in $C H_{n}\left(X \times T_{j} ; R\right)$ as a sum of cycles $A_{j r}$ supported on $X \times T_{j r}$, for $r=1, \ldots, m$, and a cycle $B_{j}$ supported on $X \times Z$. The generic fiber of $A_{j r}$ by the second projection is an $(n-j)$-cycle on $X_{k\left(T_{j r}\right)}$. By our assumption (5), this cycle is rationally equivalent to the sum of a cycle on $S_{k\left(T_{j r}\right)}$ and a cycle coming from an $(n-j)$-cycle on $X$. Therefore, $A_{j r}$ is equivalent to a sum of cycles supported on $X \times Y$ for subvarieties $Y$ of dimension at most $j-1$ and cycles supported on $W \times X$ for closed subsets $W \subsetneq X$ (using that $j>0$ ). This proves the inductive step: $[\Delta]$ in $C_{0}\left(X_{k(X)} ; R\right)$ is the image of a zero-cycle on $\left(T_{j-1}\right)_{k(X)}$ for some closed subset $T_{j-1}$ of dimension at most $j-1$ in $X$.

At the end of the induction, we have a zero-dimensional closed subset $T_{0}$ of $X$ such that the class of $\Delta$ in $C H_{0}\left(X_{k(X)} ; R\right)$ is the image of a zero-cycle $\alpha_{0}$ on $\left(T_{0}\right)_{k(X)}$. Here $T_{0}$ is a finite union of closed points, which are isomorphic to $\operatorname{Spec}(E)$ for finite extension fields $E$ of $k$. Because $X$ is geometrically integral, $(\operatorname{Spec}(E))_{k(X)}=$ $\operatorname{Spec}\left(E \otimes_{k} k(X)\right)$ is the spectrum of a field. So $C H_{0}\left(T_{0} ; R\right) \rightarrow C H_{0}\left(\left(T_{0}\right)_{k(X)} ; R\right)$ is an isomorphism. We conclude that the class of $\Delta$ in $C H_{0}\left(X_{k(X)} ; R\right)$ is in the image of $C H_{0}(X ; R)$. This gives a decomposition of the diagonal

$$
\Delta=X \times \alpha+B
$$

in $C H_{n}\left(X \times_{k} X ; R\right)$, where $\alpha$ is a zero-cycle on $X$ and $B$ is a cycle supported on $W \times X$ for some closed subset $W \subsetneq X$. This implies statement (2), by the same argument used to show that (1) implies (2). Thus all the conditions are equivalent.

We now strengthen Theorem 2.1 in a certain direction: if a variety over a field $k$ has nontrivial unramified cohomology, then its Chow groups over extension fields of $k$ have arbitrarily large cardinality (Lemma 2.5). Our proof uses the language of birational motives. One could also give a more bare-hands argument. 
Lemma 2.4. Let $k$ be a field and $R$ a commutative ring. Let $W_{1}$ be a variety, $W_{2}$ a smooth proper variety, and $X$ a separated scheme of finite type over $k$. For any integer $r$, there is a natural pairing

$$
C H_{0}\left(\left(W_{2}\right)_{k\left(W_{1}\right)} ; R\right) \otimes_{R} C H_{r}\left(X_{k\left(W_{2}\right)} ; R\right) \rightarrow C H_{r}\left(X_{k\left(W_{1}\right)} ; R\right)
$$

which agrees with the obvious pullback when the 0-cycle on $\left(W_{2}\right)_{k\left(W_{1}\right)}$ is the one associated to a dominant rational map $W_{1} \rightarrow W_{2}$. As a result, the assignment $W \mapsto C H_{r}\left(X_{k(W)} ; R\right)$ for smooth proper varieties $W$ over $k$ extends to a contravariant functor on the category of birational motives over $k$ with $R$ coefficients.

Proof. Let $M$ be an $R$-linear cycle module over $k$. The unramified cohomology group $A^{0}(W ; M)$ is defined in Rost [46, section 5] for $k$-varieties $W$. For $W$ smooth proper over $k, A^{0}(W ; M)$ is a birational invariant of $W$, which coincides with $M(k(W))_{\mathrm{nr}}$ [46, section 12]. Rost observed that unramified cohomology $A^{0}(W ; M)$ for smooth proper varieties $W$ over $k$ is a contravariant functor on the category of birational motives over $k$ [32, Theorem RC.9]. More precisely, there is a pairing

$$
C H_{0}\left(\left(W_{2}\right)_{k\left(W_{1}\right)} ; R\right) \otimes_{R} A^{0}\left(W_{2} ; M\right) \rightarrow A^{0}\left(W_{1} ; M\right)
$$

for any variety $W_{1}$ and any smooth proper variety $W_{2}$ over $k$.

It remains to observe that for a separated scheme $X$ of finite type over $k$ and an integer $r$, there is a cycle module $M$ over $k$ with $A^{0}(W ; M,-r) \cong C H_{r}\left(X_{k(W)} ; R\right)$ for all $k$-varieties $W$. (The index $-r$ refers to the grading of a cycle module, as in [46, section 5].) Namely, let $M(F)$ be the $R$-linear cycle module $A_{r}\left(X_{F} ; K_{*}\right)$, in the notation of [46, section 7]. Here $F$ runs over fields $F / k$, and $K_{*}$ denotes Milnor $K$-theory tensored with $R$. Define the grading of $M(F)$ by saying that elements of $M(F, j)$ are represented by elements of Milnor $K_{r+j}$ of function fields of $r$-dimensional subvarieties of $X_{F}$. Then, by definition,

$$
A^{0}(X ; M)=\operatorname{ker}\left(A_{r}\left(Y_{k(X)}, K_{*}\right) \rightarrow \oplus_{x \in X^{(1)}} A_{r}\left(Y_{k(x)} ; K_{*}\right)\right) .
$$

The group $A_{r}\left(Y_{k(X)} ; K_{*}, R\right)$ is the Chow group $C H_{r}\left(Y_{k(X)} ; R\right)$. The boundary map takes this graded piece of $A_{r}\left(Y_{k(X)} ; K_{*}\right)$ to a zero group (involving $K_{-1}$ of function fields of $r$-dimensional subvarieties of $Y_{k(x)}$ for codimension-1 points $x$ in $X$ ). So $A^{0}(X ; M,-r) \cong C H_{r}\left(Y_{k(X)} ; R\right)$, as we want.

Lemma 2.5. Let $X$ be a separated scheme of finite type over an algebraically closed field $k$ of characteristic zero, $R$ a commutative ring, and $r$ an integer. Suppose that there is a field $E / k$ such that $C H_{r}(X ; R) \rightarrow C H_{r}\left(X_{E} ; R\right)$ is not surjective. Then $\mathrm{CH}_{r}\left(X_{F} ; R\right)$ can have arbitrarily large cardinality for fields $F / k$. In particular, there is a field $F / k$ with $C H_{r}\left(X_{F} ; R\right)$ not finitely generated as an $R$-module.

Proof. We can assume that the field $E$ is finitely generated over $k$. Then $E$ is the function field of some variety $W$ over $k$. Since $k$ has characteristic zero, we can assume that $W$ is smooth and projective over $k$. Since $k$ is algebraically closed, $W$ is geometrically integral, and so all powers $W^{n}$ are varieties over $k$. Also, since $k$ is algebraically closed, $W$ has a 0 -cycle of degree 1 , which we can use to give a splitting $M_{\text {bir }}(W) \cong M_{\text {bir }}(k) \oplus T$ for some birational motive $T$. So, for any natural number $n, M_{\text {bir }}\left(W^{n}\right) \cong\left(M_{\text {bir }}(k) \oplus T\right)^{\otimes n}$, which contains $M_{\text {bir }}(k) \oplus T^{\oplus n}$ as a summand. By 
Lemma 2.4, it follows that for any separated $k$-scheme $X$ of finite type, we have a canonical splitting

$$
C H_{r}\left(X_{k\left(W^{n}\right)} ; R\right) \cong C H_{r}(X ; R) \oplus\left(C H_{r}\left(X_{k(W)} ; R\right) / C H_{r}(X ; R)\right)^{\oplus n} \oplus \text { (something). }
$$

For any set $S$, let $F$ be the direct limit of the function fields of the varieties $W^{T}$ over all finite subsets $T$ of $S$. Then $C H_{r}\left(X_{F} ; R\right)$ is the direct limit of the Chow groups of the varieties $X_{k\left(W^{T}\right)}$. By the previous paragraph, $C H_{r}\left(X_{F} ; R\right)$ contains a direct sum of copies of $C H_{r}\left(X_{k(W)} ; R\right) / C H_{r}(X ; R)$ indexed by the elements of $S$. Since we assumed that $C H_{r}\left(X_{k(W)} ; R\right) / C H_{r}(X ; R)$ is not zero, $C H_{r}\left(X_{F} ; R\right)$ can have arbitrarily large cardinality for fields $F / k$.

\section{Failure of the weak Chow Künneth property for finite groups}

We apply Theorem 2.1 to give the first counterexamples to the Chow Künneth property for the classifying space of a finite group $G$ over an algebraically closed field $k$, answering a question from [53, section 6] and [55, Chapter 17]. Namely, if the unramified cohomology of $B G$ is nontrivial, then the weak Chow Künneth property fails, meaning that there is a finitely generated field $F$ over $k$ with $C H^{*} B G_{k} \rightarrow C H^{*} B G_{F}$ not surjective. Examples where $B G$ has nontrivial unramified $H^{2}$ were constructed by Saltman and Bogomolov [7. Correcting Bogomolov's earlier statements, Hoshi, Kang, and Kunyavskii gave examples of groups of order $p^{5}$ for every odd prime $p$, and groups of order $2^{6}$, with nontrivial unramified $H^{2}$ [25. Theorem 1.13]. These results are sharp for all prime numbers $p$. Indeed, $p$-groups of order at most $p^{4}$ satisfy the weak Chow Künneth property [55, Theorem 11.1, Theorem 17.4], as do all groups of order 32 (Theorem 10.1).

$\mathrm{Chu}$ and Kang showed that for any $p$-group $G$ of order at most $p^{4}$ and exponent $e$, if $k$ is a field of characteristic not $p$ which contains the eth roots of unity, then $B G$ is stably rational over $k[13$. (Concretely, this means that the variety $V / G$ is stably rational over $k$ for every faithful representation $V$ of $G$ over $k$. The stable birational equivalence class of $V / G$ for a faithful representation $V$ of a finite group $G$ is independent of the representation $V$, by Bogomolov and Katsylo [6].) For 2-groups of order at most $2^{5}, B G$ is again stably rational, by $\mathrm{Chu}, \mathrm{Hu}$, Kang, and Prokhorov [12]. It is striking that $B G$ has the weak Chow Künneth property for $p$-groups of order at most $p^{4}$, and for groups of order 32 , although there is no obvious implication between stable rationality of $B G$ and the weak Chow Künneth property for $B G$. (If $B G$ can be approximated by quotients $(V-S) / G$ which are linear schemes in the sense of section 5, then both properties hold; and both properties imply the triviality of unramified cohomology.)

We show in Corollary 3.2 that for every finite group $G$ such that $B G_{k}$ has nontrivial unramified cohomology with $\mathbf{F}_{p}$ coefficients, there is an extension field $F$ of $k$ such that $C H^{i}\left(B G_{F}\right) / p$ is infinite for some $i$. This answers another question from [55, Chapter 18]. In particular, the ring $C H^{*}\left(B G_{F}\right) / p$ is not noetherian, and does not consist of transferred Euler classes of representations.

We can still ask whether the abelian group $C H^{i} B G_{F}$ is finitely generated for every finite group $G$ and every integer $i$ when $F$ is an algebraically closed field. The question of finiteness is also interesting for other classes of fields, such as finitely 
generated fields over $\mathbf{Q}$ or $\mathbf{F}_{p}$. The "motivic Bass conjecture" [30, Conjecture 37] would imply that the Chow groups of every variety over a finitely generated field are finitely generated; that would imply that each group $C H^{i} B G_{F}$ is finitely generated for every affine group scheme $G$ over a finitely generated field $F$.

Finding that the Chow Künneth property fails should be just the beginning. Let $G$ be a group of order $p^{5}$ such that $B G$ has nontrivial unramified cohomology. What is the Chow ring of $B G$ over an arbitrary field (say, containing $\overline{\mathbf{Q}}$ )? We know that it will depend on the field.

Corollary 3.1. Let $G$ be an affine group scheme of finite type over a field $k$. Suppose that $k$ is perfect and $k$ admits resolution of singularities (for example, $k$ could be any field of characteristic zero). Let $p$ be a prime number which is invertible in $k$. Suppose that the homomorphism $H^{i}(k, M) \rightarrow H_{n r}^{i}(k(V / G), M)$ of unramified cohomology is not an isomorphism, for some finite Gal $\left(k^{s} / k\right)$-module $M$ over $\mathbf{F}_{p}$, some generically free representation $V$ of $G$ over $k$, and some integer $i$. (The stable birational equivalence class of $V / G$ for $V$ generically free is independent of the representation $V$, and so this hypothesis does not depend on the choice of $V$.) Then the weak Chow Künneth property with $\mathbf{F}_{p}$ coefficients fails for $B G$ over $k$, meaning that $C H^{*}(B G) / p \rightarrow C H^{*}\left(B G_{F}\right) / p$ is not surjective for some finitely generated field $F$ over $k$.

To relate the $p$-groups mentioned earlier to this statement, note that those groups $G$ (of order $p^{5}$ for $p$ odd or order $2^{6}$ for $p=2$ ) are shown to have nontrivial unramified Brauer group $H_{\mathrm{nr}}^{2}\left(k(V / G), G_{m}\right)$, where $k$ is an algebraically closed field $k$ in which $p$ is invertible. This group is $p$-power torsion, by a transfer argument. By results of Grothendieck, $H_{\mathrm{nr}}^{2}\left(k(V / G), \mu_{p}\right)$ is isomorphic to the $p$-torsion subgroup of $H_{\mathrm{nr}}^{2}\left(k(V / G), G_{m}\right)$ [17, Proposition 4.2.3]. Therefore $H_{\mathrm{nr}}^{2}\left(k(V / G), \mu_{p}\right)$ is also nonzero, and so Corollary 3.1 applies to these groups $G$.

Explicitly, for any prime number $p \geq 5$, here is an example of a group $G$ of order $p^{5}$ with unramified $H^{2}$ (over $\mathbf{C}$ ) not zero [25, proof of Theorem 2.3]. In this presentation, we use the notation $[g, h]=g^{-1} h^{-1} g h$.

$$
\begin{aligned}
& G=\left\langle f_{1}, f_{2}, f_{3}, f_{4}, f_{5}\right| f_{i}^{p}=1 \text { for all } i, f_{5} \text { central, } \\
& \left.\quad\left[f_{2}, f_{1}\right]=f_{3},\left[f_{3}, f_{1}\right]=f_{4},\left[f_{4}, f_{1}\right]=\left[f_{3}, f_{2}\right]=f_{5},\left[f_{4}, f_{2}\right]=\left[f_{4}, f_{3}\right]=1\right\rangle
\end{aligned}
$$

Proof. (Corollary 3.1) By definition, $C H^{i} B G$ is isomorphic to $C H^{i}(V-S) / G$ for any representation $V$ of $G$ over $k$ and any $G$-invariant (Zariski) closed subset $S$ such that $G$ acts freely on $V-S$ with quotient a scheme and $S$ has codimension greater than $i$ in $V$ [53, Theorem 1.1], [55, Theorem 2.5]. By the basic exact sequence for equivariant Chow groups, the homomorphism

$$
C H^{*} B G=C H_{G}^{*} V \rightarrow C H_{G}^{*}(V-S)=C H^{*}(V-S) / G
$$

is surjective [19, Proposition 5], [55, Lemma 2.9].

Suppose that $C H^{*}(B G) / p \rightarrow C H^{*}\left(B G_{F}\right) / p$ is surjective for every finitely generated field $F$ over $k$. Let $V$ be a representation of $G$ with a closed subset $S \subsetneq V$ such that $G$ acts freely on $V-S$ with quotient a separated scheme $U=(V-S) / G$. By the previous paragraph, applied to $G$ and $G_{F}$, it follows that $C H^{*}(U) / p \rightarrow C H^{*}\left(U_{F}\right) / p$ is surjective for every finitely generated field $F$ over $k$. By Corollary 2.2 , $U$ has the 
birational motive of a point, with $\mathbf{F}_{p}$ coefficients. It follows that the field $k(U)$ over $k$ has trivial unramified cohomology with coefficients in any $\mathbf{F}_{p}$-linear cycle module over $k$. Galois cohomology (with $p$ invertible in $k$, as we assume) is an example of a cycle module. Explicitly, for any finite $\operatorname{Gal}\left(k_{s} / k\right)$-module $M$ killed by $p$, the assignment $F \mapsto \oplus_{i} H^{i}\left(F, M \otimes \mu_{p}^{\otimes i}\right)$ for finitely generated fields $F$ over $k$ is a cycle module over $k$ [46, Remark 2.5]. That completes the proof.

The following corollary strengthens Corollary 3.1. We give the first examples of finite groups $G$ and prime numbers $p$ such that the Chow group $C H^{i}\left(B G_{F}\right) / p$ is infinite, for some $i$ and some field $F$. Namely, we can take a group of order $p^{5}$ for $p$ odd, or of order $2^{6}$, with nontrivial unramified cohomology.

Corollary 3.2. Let $G$ be a finite group, and let $p$ be a prime number. Suppose that the unramified cohomology $H_{n r}^{i}\left(\overline{\mathbf{Q}}(V / G), \mathbf{F}_{p}\right)$ is not zero, for some generically free representation $V$ of $G$ over $\overline{\mathbf{Q}}$ and some $i>0$. Then there is a field $F$ containing $\overline{\mathbf{Q}}$ and a positive integer $r$ such that $C H^{r}\left(B G_{F}\right) / p$ is infinite. It follows that the ring $C H^{*}\left(B G_{F}\right) / p$ is not noetherian.

Proof. Corollary 3.1 gives an extension field $E$ of $\overline{\mathbf{Q}}$ such that $C H^{r}(B G) / p \rightarrow$ $C H^{r}\left(B G_{E}\right) / p$ is not surjective for some $r$. So, for a finite-dimensional approximation $U=(V-T) / G$ to $B G$ with $T$ of codimension greater than $r, C H^{r}(U) / p \rightarrow$ $C H^{r}\left(U_{E}\right) / p$ is not surjective. By Lemma 2.5, there is a field $F / \overline{\mathbf{Q}}$ with $C H^{r}\left(U_{F}\right) / p$ infinite. Equivalently, $C H^{r}\left(B G_{F}\right) / p$ is infinite. Since $C H^{*}\left(B G_{F}\right) / p$ is a graded

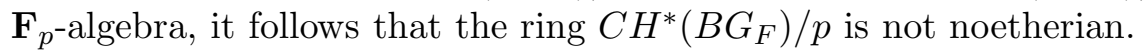

\section{The weak Chow Künneth property for smooth proper $k$-schemes}

In this section, we characterize the smooth proper $k$-schemes whose Chow groups remain unchanged under arbitrary field extensions: they are the schemes whose Chow motive is a Tate motive. This type of result for smooth proper $k$-schemes has a long history, including results by Bloch [35, Proposition 3.12], 4, Appendix to Lecture 1], Bloch-Srinivas [5, Jannsen [28, Theorem 3.5], and Kimura 34. Shinder gave a convenient version of Bloch's argument [48. One difference from most earlier results is that we consider Chow groups with coefficients in any commutative ring, not just the rational numbers.

In the rest of the paper, Theorem 4.1 is used only to prove Corollary [7.3. Nonetheless, the proof, using the diagonal cycle, helped to suggest the proof of Theorem 7.2 about arbitrary schemes. Theorem 2.1 is a "birational analog" of Theorem 4.1; in particular, the equivalent properties in Theorem 4.1 are not birationally invariant.

Theorem 4.1. Let $M$ be a Chow motive over a field $k$ with coefficients in a commutative ring $R$. (For example, $M$ could be the motive $M(X)$ for a smooth proper $k$-scheme $X$.) Suppose that $M$ has the weak Chow Künneth property, meaning that the morphism $C H_{*}(M) \rightarrow C H_{*}\left(M_{F}\right)$ is a surjection of $R$-modules for every finitely generated field $F / k$. Then $M$ is a summand of a finite direct sum of Tate motives $R(j)[2 j]$ for integers $j$. 
Conversely, suppose that a Chow motive $M$ is a summand of a finite direct sum of Tate motives. Then $C H_{*}(M) \rightarrow C H_{*}\left(M_{F}\right)$ is an isomorphism for every field $F / k$, and $M$ has the Chow Künneth property that $C H_{*}(M) \otimes_{R} C H_{*}(Y ; R) \rightarrow$ $C H_{*}\left(M \otimes M^{c}(Y)\right)$ is an isomorphism of $R$-modules for every separated $k$-scheme $Y$ of finite type. Also, $C H_{*}(M)$ is a finitely generated projective $R$-module, and $C H_{*}(M) \cong H_{*}\left(M_{\mathbf{C}}, R\right)$ if there is an embedding $k \hookrightarrow \mathbf{C}$. Finally, $M$ has the Künneth property for motivic homology in the sense that

$$
C H_{*}(M) \otimes_{R} H_{*}^{M}(Y, R(*)) \cong H_{*}^{M}\left(M \otimes M^{c}(Y), R(*)\right)
$$

for every separated $k$-scheme $Y$ of finite type.

The notation $M^{c}(Y)$ is suggested by Voevodsky's triangulated category of motives (discussed in section 5), but below we say explicitly what this means.

If $R$ is a PID, then the conditions in the theorem are also equivalent to $M$ being a finite direct sum of Tate motives (without having to take a direct summand). For an arbitrary commutative ring $R$, it is essential to allow direct summands.

The conclusion cannot be strengthened to say that $X$ is a linear scheme or a rational variety. There are Barlow surfaces over $\mathbf{C}$ whose Chow motive with $\mathbf{Z}$ coefficients is a direct sum of Tate motives, for example by Theorem 4.1 and 2 , Proposition 1.9]. It follows that these smooth projective surfaces have the Chow Künneth property, although they are of general type and hence not rational.

Before proving Theorem 4.1, let us define the category of Chow motives over $k$ with coefficients in $R$. To agree with the conventions in Voevodsky's triangulated category of motives $D M(k ; R)$ (section 5 ), we think of the basic functor $X \mapsto M(X)$ from smooth proper $k$-schemes to Chow motives as being covariant, and we write the motive of $\mathbf{P}_{k}^{1}$ as $R \oplus R(1)[2]$. Covariance is only a minor difference from the conventions in Scholl's paper [47, because the category of Chow motives is selfdual. (The "shift" [2] is written in order to agree with the notation in $D M(k ; R)$; it has no meaning by itself in the category of Chow motives.) We will only consider $D M(k ; R)$ when the exponential characteristic of $k$ is invertible in $R$; in that case, the category of Chow motives is equivalent to a full subcategory of $D M(k ; R)$.

For smooth proper varieties $X$ and $Y$ over $k$, define the $R$-module of correspondences of degree $r$ from $X$ to $Y$ as

$$
\operatorname{Corr}_{r}(X, Y)=C H_{\operatorname{dim}(X)+r}\left(X \times_{k} Y ; R\right) .
$$

We extend this definition to all smooth proper $k$-schemes by taking direct sums. For smooth proper $k$-schemes $X, Y, Z$, there is a composition of correspondences

$$
\operatorname{Corr}_{r}(X, Y) \otimes_{R} \operatorname{Corr}_{s}(Y, Z) \rightarrow \operatorname{Corr}_{r+s}(X, Z),
$$

written as $f \otimes g \mapsto g f$, given by pulling back the two cycles from $X \times Y$ and $Y \times Z$ to $X \times Y \times Z$, multiplying, and pushing forward to $X \times Z$.

A Chow motive over $k$ with coefficients in $R$, written $(M(X)(a)[2 a], p)$, consists of a smooth proper $k$-scheme $X$, an integer $a$, and an idempotent $p=p^{2}$ in $\operatorname{Corr}_{0}(X, X)$. The morphisms of Chow motives are given by

$$
\operatorname{Hom}((M(X)(a)[2 a], p),(M(Y)(b)[2 b], q))=q \operatorname{Corr}_{a-b}(X, Y) p \subset \operatorname{Corr}_{a-b}(X, Y)
$$


Composition of correspondences makes the Chow motives over $k$ into a category. We write $M(X)$ for the motive $(M(X)(0)[0], \Delta)$, where $\Delta$ is the diagonal in $X \times_{k} X$. Thus $X \mapsto M(X)$ is a covariant functor from smooth proper $k$-schemes to Chow motives. The Tate motive $R(a)[2 a]$ is $M(\operatorname{Spec}(k))(a)[2 a]$. Define the Chow groups of a motive $M$ by $C H_{a}(M)=\operatorname{Hom}(R(a)[2 a], M)$; then the group $C H_{a}(M(X))$ is isomorphic to the usual Chow group $C H_{a}(X ; R)$ of a smooth proper $k$-scheme $X$.

The category of Chow motives is symmetric monoidal, with tensor product $\otimes$ such that $M(X) \otimes M(Y) \cong M\left(X \times_{k} Y\right)$ for smooth proper $k$-schemes $X$ and $Y$. There is an involution $M \mapsto M^{*}$ on Chow motives, defined on objects by

$$
(M(X)(a)[2 a], p)^{*}=\left(M(X)(-n-a)[-2 n-2 a], p^{t}\right)
$$

for $X$ of pure dimension $n$. It is immediate that the natural morphism $M \rightarrow M^{* *}$ is an isomorphism, and that

$$
\operatorname{Hom}(M \otimes N, P) \cong \operatorname{Hom}\left(M, N^{*} \otimes P\right)
$$

for all Chow motives $M, N, P$ [47, section 1.1.5]. That is, the category of Chow motives is a rigid additive tensor category, with internal Hom given by $\underline{\operatorname{Hom}}(M, N)=$ $M^{*} \otimes N$. For a field extension $F / k$, there is an obvious functor from Chow motives over $k$ to Chow motives over $F$, taking $M(X)$ to $M\left(X_{F}\right)$ for smooth proper $k$-schemes $k$.

As an extension of the previous notation, for any Chow motive $M=(M(X)(a)[2 a], p)$ over $k$ and any $k$-scheme $Y$ of finite type, we define the Chow groups $C H_{*}(M \otimes$ $\left.M^{c}(Y)\right)$ as the summand of the Chow groups $C H_{*}\left(X \times_{k} Y ; R\right)$ given by $p$. (At this point, $M^{c}(Y)$ has no meaning by itself. In section 5, $M^{c}(Y)$ will be used to denote the compactly supported motive of $Y$ in the triangulated category of motives $D M(k ; R)$.

Proof. (Theorem 4.1) Let $M$ be a Chow motive which has the weak Chow Künneth property, meaning that $C H_{*} M \rightarrow C H_{*}\left(M_{F}\right)$ is surjective for all finitely generated fields $F$ over $k$. Then the $R$-linear map $C H_{*} M \otimes_{R} C H_{*} Y \rightarrow C H_{*}\left(M \otimes M^{c}(Y)\right)$ is surjective for every $k$-scheme $Y$ of finite type. (In this proof, we write $C H_{*}(Y)$ to mean $C H_{*}(Y ; R)=C H_{*}(Y) \otimes_{\mathbf{Z}} R$.) To prove this, do induction on the dimension of $Y$, using the commutative diagram of exact sequences for any closed subscheme $S$ of $Y$ :

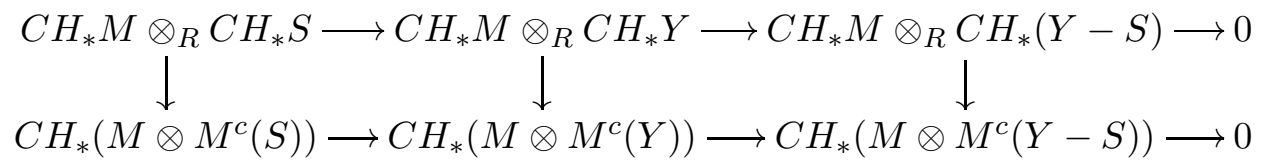

Here we use that, for a $k$-variety $Y, C H_{*}\left(M_{k(Y)}\right)=\lim _{\longrightarrow} C H_{*}\left(M \otimes M^{c}(Y-S)\right)$,

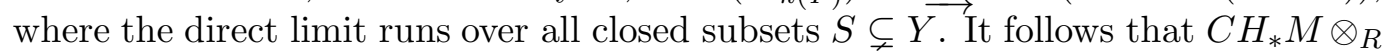
$C H_{*} N \rightarrow C H_{*}(M \otimes N)$ is surjective for all Chow motives $N$.

For any Chow motives $N$ and $P$, we have $\operatorname{Hom}(N, P)=\operatorname{Hom}(R \otimes N, P)=$ $\operatorname{Hom}(R, \underline{\operatorname{Hom}}(N, P))$. By Lemma 5.5, the identity map on the Chow motive $M$ corresponds to an element $1_{M} \in \operatorname{Hom}\left(R, M^{*} \otimes M\right)=C H_{0}\left(M^{*} \otimes M\right)$. (When $M$ is the motive of a smooth proper variety $X, 1_{M}$ is the class of the diagonal on $X \times X$.)

For the given motive $M$, we showed that $C H_{*} M \otimes_{R} C H_{*} N \rightarrow C H_{*}(M \otimes N)$ is surjective for all Chow motives $N$, and we apply this to $N=M^{*}$. So we can 
write $1_{M}=\sum_{i=1}^{r} \alpha_{i} \otimes \beta_{i}$ in $C H_{0}\left(M^{*} \otimes M\right)$ for some $\alpha_{1}, \ldots, \alpha_{r} \in C H_{*}\left(M^{*}\right)$ and $\beta_{1}, \ldots, \beta_{r} \in C H_{*} M$. Here $\alpha_{i}$ is in $C H_{-b_{i}}\left(M^{*}\right)$ and $\beta_{i}$ is in $C H_{b_{i}} M$ for some integers $b_{1}, \ldots, b_{r}$. Let $N=\oplus_{i=1}^{r} R\left(b_{i}\right)\left[2 b_{i}\right]$. Then $\left(\beta_{1}, \ldots, \beta_{r}\right)$ can be viewed as a morphism $\beta: N \rightarrow M$, and $\left(\alpha_{1}, \ldots, \alpha_{r}\right)$ can be viewed as a morphism $N^{*} \rightarrow M^{*}$, or equivalently $\alpha: M \rightarrow N$. The equation $1_{M}=\sum \alpha_{i} \otimes \beta_{i}$ in $C H_{0}\left(M^{*} \otimes M\right)$ means that the composition $M \rightarrow N \rightarrow M$ is the identity. Since idempotents split in the category of Chow motives, it follows that $M$ is a direct summand of $N$, which is a finite direct sum of Tate motives. One direction of the theorem is proved.

The converse statements in the theorem are clear for a finite direct sum of Tate motives. That implies the converse statements for any summand of a finite direct sum of Tate motives.

\section{The triangulated category of motives}

This section summarizes the properties of Voevodsky's triangulated category of motives over a field $k, D M(k ; R)$. Every separated scheme of finite type over $k$ (not necessarily smooth and proper) determines an object in this category, and Chow groups are given by morphisms from a fixed object (a Tate motive) in this category. So $D M(k ; R)$ is a natural setting for studying Chow groups of $k$-schemes that need not be smooth and proper.

We use the triangulated category of motives for at least two purposes in this paper. First, we need it even to state the characterization of those schemes of finite type which satisfy the Künneth property for motivic homology groups (Theorem 7.2). The corresponding characterization for smooth proper schemes (Theorem 4.1) used only the more elementary category of Chow motives. Second, we need the triangulated category of motives in order to define the motive $M^{c}(B G)$ of a classifying space and to study when that motive is mixed Tate (sections 8 and 9).

Let $k$ be a field. Thanks to recent developments in the theory of motives, $k$ need not be assumed to be perfect or to admit resolution of singularities. We put one restriction on the coefficient ring $R$, as follows. The exponential characteristic of $k$ means 1 if $k$ has characteristic zero, or $p$ if $k$ has characteristic $p>0$. For the rest of this section, we assume that the exponential characteristic of $k$ is invertible in $R$. This assumption is used to prove the basic properties of the compactly supported motive of a scheme $X$ over $k, M^{c}(X)$, such as the localization triangle. (This assumption can be avoided when we know resolution of singularities over $k$.) This assumption on $R$ should be understood throughout the paper when we discuss motives $M^{c}(X)$.

A readable introduction to Voevodsky's triangulated categories of motives over $k$ is [56]. Let $R$ be a commutative ring. We primarily use the "big" triangulated category $D M(k ; R)$ of motives with coefficients in $R$, which contains the direct sum of an arbitrary set of objects. Also, the motive $R(1)$ is invertible in $D M(k ; R)$, as discussed below. (Voevodsky originally considered the subcategory $D M_{-}^{\text {eff }}(k)$ of "bounded above effective motives", which does not have arbitrary direct sums.) Following Cisinski and Déglise, $D M(k ; R)$ is defined to be the homotopy category of $G_{m}^{\mathrm{tr}}$-spectra of (unbounded) chain complexes of Nisnevich sheaves with transfers which are $A^{1}$-local [45, section 2.3], [14, Example 6.25]. For $k$ perfect, Röndigs and Østvær showed that the category $D M(k ; \mathbf{Z})$ is equivalent to the homotopy 
category of modules over the motivic Eilenberg-MacLane spectrum $H \mathbf{Z}$ in MorelVoevodsky's stable homotopy category $S H(k)$ [45, Theorem 1]. This is an analog of the equivalence between the derived category $D(\mathbf{Z})$ of abelian groups and the homotopy category of modules over the Eilenberg-MacLane spectrum $H \mathbf{Z}$ in the category of spectra in topology [21, Theorem 8.9].

Let $k^{\text {perf }}$ denote the perfect closure of $k$. That is, $k^{\text {perf }}$ is equal to $k$ if $k$ has characteristic zero, and $k^{\text {perf }}$ consists of all $p^{r}$ th roots of elements of $k$ for all $r \geq 0$ if $k$ has characteristic $p>0$. Under our assumption that $p$ is invertible in $R$, Cisinski and Déglise proved the following convenient result, following a suggestion by Suslin [16, Proposition 8.1].

Theorem 5.1. The pullback functor $D M(k ; R) \rightarrow D M\left(k^{\text {perf }} ; R\right)$ is an equivalence of categories.

By Theorem 5.1, most results on motives which previously assumed that $k$ is perfect immediately generalize to an arbitrary field $k$, given our assumption that the exponential characteristic of $k$ is invertible in $R$. We will mention some examples in what follows.

By definition of a triangulated category (such as $D M(k ; R)$ ), every morphism $X \rightarrow Y$ fits into a distinguished triangle $X \rightarrow Y \rightarrow Z \rightarrow X[1]$. Here $Z$ is called a cone of the morphism $X \rightarrow Y$. It is unique up to isomorphism, but not (in general) up to unique isomorphism.

There are two natural functors from schemes to motives, which we write as $M(X)$ and $M^{c}(X)$. These were defined by Voevodsky when $k$ is a perfect field which admits resolution of singularities (as we know for $k$ of characteristic zero) [56, section 2.2]. Kelly extended these constructions to any perfect field $k$, under our assumption that the exponential characteristic of $k$ is invertible in $R$, using Gabber's work on alterations [33, Lemmas 5.5.2 and 5.5.6]. Finally, these constructions now apply to any field $k$ : given a separated scheme $X$ of finite type over $k$, we have objects $M\left(X_{k_{\text {perf }}}\right)$ and $M^{c}\left(X_{k^{\text {perf }}}\right)$ of $D M\left(k^{\text {perf }} ; R\right)$ by Kelly, hence objects $M(X)$ and $M^{c}(X)$ of $D M(k ; R)$ by Theorem 5.1 .

In more detail, there is a covariant functor $X \mapsto M(X)$ from the category of separated schemes of finite type over $k$ to $D M(k ; R)$. Also, there is a covariant functor $X \mapsto M^{c}(X)$ (the motive of $X$ "with compact support") from the category of separated schemes of finite type and proper morphisms to $D M(k ; R)$. A flat morphism $X \rightarrow Y$ determines a pullback map $M^{c}(Y) \rightarrow M^{c}(X)$. The motives $M(X)$ and $M^{c}(X)$ are isomorphic for $X$ proper over $k$.

The category $D M(k ; R)$ has objects called $R(j)$ for all integers $j$. The motives $R(j)[2 j]$ are called the Tate motives. One interpretation of Tate motives is that $M^{c}\left(A_{k}^{j}\right)=R(j)[2 j]$ for $j \geq 0$. More generally, for an affine bundle $Y \rightarrow X$ (a morphism that is locally on $X$ isomorphic to a product with affine space $A^{r}$ ), we have the homotopy invariance statements that $M(Y) \cong M(X)$, whereas $M^{c}(Y) \cong$ $M^{c}(X)(r)[2 r]$.

The category $D M(k ; R)$ is a tensor triangulated category, with a symmetric monoidal product $\otimes\left[14\right.$, Example 6.25]. We have $M(X) \otimes M(Y)=M\left(X \times_{k} Y\right)$ and $M^{c}(X) \otimes M^{c}(Y)=M^{c}\left(X \times_{k} Y\right)$ for $k$-schemes $X$ and $Y$ [56, Proposition 4.1.7], [33, Proposition 5.5.8]. The motive $R=R(0)$ of a point is the identity object for the tensor product. The motive $R(1)$ is invertible in the sense that $R(a) \otimes R(b) \cong R(a+b)$ for all integers $a$ and $b$. 
The category $D M(k ; R)$ has internal Hom objects, with natural isomorphisms

$$
\operatorname{Hom}(A \otimes B, C) \cong \operatorname{Hom}(A, \underline{\operatorname{Hom}}(B, C))
$$

for all motives $A, B, C$. Moreover, the internal Hom preserves distinguished triangles in each variable, up to a sign change in the boundary map [26, Definition 6.6.1, Theorem 7.1.11]. (All this is part of Cisinski-Déglise's result that $S \mapsto D M(S ; R)$ is a "premotivic category" for finite-dimensional noetherian schemes $S$ [15, section 11.1.2].) It follows that, for any motive $B$ in $D M(k ; R)$, the functor $\cdot \otimes B$ is a left adjoint, and therefore preserves arbitrary direct sums.

To understand the two functors, note that the Chow groups $C H_{i} X$ are determined by $M^{c}(X)$, whereas Chow cohomology groups $C H^{i} X$ for $X$ smooth over $k$ are determined by $M(X)$. Namely,

$$
C H_{i}(X) \otimes \mathbf{z} R=\operatorname{Hom}\left(R(i)[2 i], M^{c}(X)\right)
$$

for any separated scheme $X$ of finite type over $k$, while

$$
C H^{i}(X) \otimes_{\mathbf{z}} R=\operatorname{Hom}(M(X), R(i)[2 i])
$$

for $X$ also smooth over $k$ [56, section 2.2]. Voevodsky defined motivic cohomology and (Borel-Moore) motivic homology for any separated scheme $X$ of finite type over $k$ by

$$
H_{M}^{j}(X, R(i))=\operatorname{Hom}(M(X), R(i)[j])
$$

and

$$
H_{j}^{M}(X, R(i))=\operatorname{Hom}\left(R(i)[j], M^{c}(X)\right) .
$$

For a separated scheme $X$ of finite type over $k$ and a closed subscheme $Z$ of $X$, there is a distinguished triangle in $D M(k ; R)$, the localization triangle:

$$
M^{c}(Z) \rightarrow M^{c}(X) \rightarrow M^{c}(X-Z) \rightarrow M^{c}(Z)[1] .
$$

(This was proved by Voevodsky when $k$ is perfect and admits resolution of singularities [56, section 2.2], by Kelly for any perfect field $k$ with our assumption on $R$ [33. Proposition 5.5], and by Theorem 5.1 for an arbitrary field k.) This triangle induces a long exact sequence of motivic homology groups, called the localization sequence.

Bloch defined higher Chow groups as the homology of an explicit complex of algebraic cycles. Higher Chow groups are essentially the same as motivic homology, but (by tradition) they are numbered by codimension. Namely, for an equidimensional separated scheme $X$ of dimension $n$ over $k$,

$$
C H^{n-j}(X, i-2 j ; R) \cong H_{i}^{M}(X, R(j))
$$

(For $k$ admitting resolution of singularities and $X$ quasi-projective over $k$, this is [56, Proposition 4.2.9]. Kelly modified the argument to replace the assumption on resolution of singularities with our assumption on $R$ [33, Theorem 5.6.4]. Finally, the assumption of quasi-projectivity was needed for Bloch's proof of the localization sequence for higher Chow groups [3], but Levine has now proved the localization sequence for the higher Chow groups of all schemes of finite type over a field 37 , Theorem 0.7].) 
Some higher Chow groups are zero by the definition, because they consist of cycles of negative dimension or negative codimension. It follows that the motivic homology $H_{i}^{M}(X, R(j))$ of a separated $k$-scheme $X$ is zero unless $i \geq 2 j$ and $i \geq j$ and $j \leq \operatorname{dim}(X)$.

For any motive $A$ in $D M(k ; R)$, we define the motivic homology groups of $A$ to mean the groups $H_{j}^{M}(A, R(i))=\operatorname{Hom}(R(i)[j], A)$. Note that what we call the motivic homology groups of a separated $k$-scheme $X$ of finite type are the motivic homology groups of the motive $M^{c}(X)$, not those of $M(X)$ (although the two motives are isomorphic for $X$ proper over $k$ ).

Let $\mathcal{T}$ be a triangulated category with arbitrary direct sums. A localizing subcategory of $\mathcal{T}$ means a strictly full triangulated subcategory which is closed under arbitrary direct sums. Following Röndigs and Østvær, the triangulated category $\operatorname{DMT}(k ; R)$ of mixed Tate motives with coefficients in $R$ is the smallest localizing subcategory of $D M(k ; R)$ that contains $R(j)$ for all integers $j$ [45]. Because the tensor product $\otimes$ on $D M(k ; R)$ is compatible with distinguished triangles and with arbitrary direct sums, the tensor product of two mixed Tate motives is a mixed Tate motive.

The category of mixed Tate motives is analogous to the category of cellular spectra in the stable homotopy category $S H(k)$ studied by Voevodsky [57] and Dugger-Isaksen [18]. (Actually, Voevodsky says "T-cellular" and Dugger and Isaksen say "stably cellular".) Namely, let $T$ be the suspension spectrum of the pointed $k$-space $\left(\mathbf{P}_{k}^{1}\right.$, point); the triangulated category of cellular spectra is defined as the smallest localizing subcategory of $S H(k)$ that contains $T^{j}$ for all integers $j$.

As with motives, there are two natural functors from separated $k$-schemes $X$ of finite type to $S H(k)$ : the usual functor (which we write as $X \mapsto S(X)$ or $\left.X \mapsto \Sigma_{T}^{\infty} X^{+}\right)$and a compactly supported version, $X \mapsto S^{c}(X)$. Explicitly, for any compactification $\bar{X}$ of a $k$-scheme $X, S^{c}(X)$ is the spectrum associated to the pointed $k$-space $\bar{X} /(\bar{X}-X)$. There is a functor from $S H(k)$ to $D M(k ; R)$, which one can view as smashing with the Eilenberg-MacLane spectrum $H R$, and this takes $S(X)$ to $M(X)$ and $S^{c}(X)$ to $M^{c}(X)$. In particular, the spectrum $T$ goes to the motive $R(1)[2]$.

In a triangulated category with arbitrary direct sums, every idempotent splits [9, Proposition 3.2]. Applying this to the category of mixed Tate motives, it follows that every summand of a mixed Tate motive in $D M(k ; R)$ is a mixed Tate motive.

Let $\mathcal{T}$ be a triangulated category with arbitrary direct sums. An object $X$ of $\mathcal{T}$ is called compact if $\operatorname{Hom}(X, \cdot)$ commutes with arbitrary direct sums. The objects $M(X)(a)[b]$ and $M^{c}(X)(a)[b]$ are compact in $D M(k ; R)$ for every separated $k$-scheme $X$ of finite type [33, Lemmas 5.5.2 and 5.5.6]. A set $\mathcal{P}$ of objects generates $\mathcal{T}$ if every object $Y$ of $\mathcal{T}$ such that $\operatorname{Hom}(P[a], Y)=0$ for all objects $P$ in $\mathcal{P}$ and all integers $a$ is zero. A triangulated category $\mathcal{T}$ is compactly generated if it has arbitrary direct sums and it is generated by a set of compact objects.

The following result by Neeman helps to understand the notion of generators for a triangulated category [41, Theorem 2.1].

Lemma 5.2. Let $\mathcal{T}$ be a triangulated category with arbitrary direct sums, and let $\mathcal{P}$ be a set of compact objects. The following are equivalent:

(1) The smallest localizing subcategory of $\mathcal{T}$ that contains $\mathcal{P}$ is equal to $\mathcal{T}$.

(2) The set $\mathcal{P}$ generates $\mathcal{T}$. That is, any object $X$ in $\mathcal{T}$ with $\operatorname{Hom}(P[a], X)=0$ 
for all $P$ in $\mathcal{P}$ and $a \in \mathbf{Z}$ must be zero.

Corollary 5.3. A mixed Tate motive with zero motivic homology must be zero.

Proof. The objects $R(a)$ for integers a generate the category $D M T(k ; R)$, by Lemma 5.2. Since $H_{j}^{M}(A, R(i))=\operatorname{Hom}(R(i)[j], A)$ for a motive $A$, the corollary is proved.

Lemma 5.4. Let $k$ be a field. Then the category $D M(k ; R)$ is compactly generated, with a set of generators given by the compact objects $M(X)(a)$ for $X$ smooth projective over $k$ and $a$ an integer.

Proof. This was proved by Voevodsky when $k$ is perfect and admits resolution of singularities [56, Corollary 3.5.5]. Given our assumption that the exponential characteristic of $k$ is invertible in $R$, Kelly generalized this result to any perfect field $k$ [33, Proposition 5.5.3]. The generalization to an arbitrary field $k$ follows from Theorem 5.1 .

A reassuring fact is that if the motive $M^{c}(X)$ in $D M(k ; R)$ of a separated $k$ scheme $X$ of finite type is mixed Tate, then it is a summand of an object of the smallest strictly full triangulated subcategory of $D M(k ; R)$ that contains $R(j)$ for all integers $j$. In other words, $M^{c}(X)$ can be described by a finite diagram of objects $R(j)$. This follows from a general result about triangulated categories. Define a thick subcategory of a triangulated category to be a strictly full triangulated subcategory that is closed under direct summands. Let $\mathcal{T}$ be a compactly generated triangulated category, and let $\mathcal{P}$ be a set of compact generators. (We have in mind the category of mixed Tate motives, generated by the objects $R(j)$ for integers $j$.) Then Neeman showed that any compact object in $\mathcal{T}$ belongs to the smallest thick subcategory of $\mathcal{T}$ that contains $\mathcal{P}$ [41, Theorem 2.1].

The category $D M_{\mathrm{gm}}(k ; R)$ of geometric motives is defined as the smallest thick subcategory of $D M(k ; R)$ that contains $M(X)(a)$ for all smooth separated schemes $X$ of finite type over $k$ and all integers $a$. In fact, it suffices to use $M(X)(a)$ for smooth projective varieties $X$ over $k$ and all integers $a$, by Lemma 5.4. Another application of Neeman's theorem gives that $D M_{\mathrm{gm}}(k ; R)$ is the subcategory of all compact objects in $D M(k ; R)$.

A linear scheme over a field $k$ is defined inductively: affine space $A_{k}^{n}$ is a linear scheme for any $n \geq 0$; for any scheme $X$ of finite type over $k$ with a closed subscheme $Z$, if $Z$ and $X-Z$ are linear schemes, then $X$ is a linear scheme; and if $X$ and $Z$ are linear schemes, then $X-Z$ is a linear scheme. (A slightly narrower class of linear schemes was studied in [54].) Some examples of linear schemes are all toric varieties, not necessarily smooth or compact, the discriminant hypersurface and its complement, and many quotients of affine space by finite group actions. Linear schemes can have torsion in their Chow groups and homology groups, and they can have nonzero rational homology in odd degrees. (To talk about rational homology, assume that the base field is the complex numbers.)

From the localization triangle, a straightforward induction shows that for any linear scheme $X$ over $k$, the compactly supported motive $M^{c}(X)$ with any coefficient ring $R$ is a mixed Tate motive. Likewise, for any linear scheme $X$, the spectrum $S^{c}(X)$ is cellular in $S H(k)$. (Dugger and Isaksen asked whether the spectrum $S(X)$ is cellular for linear schemes $X$, and proved this in some examples [18, section 1.1]. 
Arguably, the more natural spectrum associated to a linear scheme $X$ is $S^{c}(X)$, which is clearly cellular. For $X$ proper over $k, S(X)$ and $S^{c}(X)$ are isomorphic.)

Let $X$ and $Y$ be smooth proper varieties over $k$. Then the set of morphisms from $M(X)$ to $M(Y)$ in $D M(k ; R)$ is the Chow group $C H_{\operatorname{dim}(X)}\left(X \times_{k} Y ; R\right)[56$, section 2.2]. Composition of morphisms $M(X) \rightarrow M(Y) \rightarrow M(Z)$ is given by the composition of correspondences. As a result, the smallest strictly full subcategory of $D M(k ; R)$ that is closed under direct summands and contains $M(X)(a)[2 a]$ for all smooth proper schemes $X$ over $k$ and all integers $a$ is equivalent to the category of Chow motives over $k$ with coefficients in $R$, as defined in section 4 .

We define $N^{*}=\underline{\operatorname{Hom}}(N, R)$. A version of Poincaré duality says that $M^{c}(X) \cong$ $M(X)^{*}(n)[2 n]$ for $X$ smooth of pure dimension $n$ over $k$ [33, Theorem 5.5.14]. The internal Hom of motives has a simple description for compact objects, as follows.

Lemma 5.5. Let $M$ be an object of $D M_{g m}(k ; R)$, for example the motive $M^{c}(X)(a)[b]$ for a scheme $X$ of finite type over $k$ and $a, b \in \mathbf{Z}$. Let $N$ be any object of $D M(k ; R)$. Then the morphism $M^{*} \otimes N \rightarrow \underline{H o m}(M, N)$ is an isomorphism.

Also, for $M$ in $D M_{g m}(k ; R)$, the natural map $M \rightarrow M^{* *}$ is an isomorphism.

Proof. At first, let $M^{*}$ denote the object $\underline{\operatorname{Hom}}_{\mathrm{gm}}(M, R)$ in the subcategory $D M_{\mathrm{gm}}(k ; R)$ of compact objects. Then Voevodsky and Kelly prove that $M \rightarrow M^{* *}$ is an isomorphism for $M$ compact, and also that $M^{*} \otimes N \rightarrow \underline{\operatorname{Hom}}_{g m}(M, N)$ is an isomorphism for $M$ and $N$ compact [56, Theorem 4.3.7], [33, Theorem 5.5.14]. That is, the map

$$
\operatorname{Hom}\left(A, B^{*} \otimes C\right) \rightarrow \operatorname{Hom}(A \otimes B, C)
$$

associated to $B^{*} \otimes B \rightarrow R$ is a bijection for all compact objects $A, B, C$.

For $A$ and $B$ compact, the map of Hom sets above turns arbitrary direct sums of motives $C$ into direct sums, and fits into long exact sequences for any distinguished triangle of objects $C$. By Lemmas $[5.2$ and [5.4, it follows that the map is an isomorphism for $A$ and $B$ compact and $C$ arbitrary. For $B$ compact and $C$ arbitrary, both Hom sets turn arbitrary direct sums of motives $A$ into products, and they fit into long exact sequences for any distinguished triangle of objects $A$. Therefore the map is an isomorphism for $B$ compact and $A$ and $C$ any motives. That is, the internal Hom in $D M(k ; R)$ has $\underline{\operatorname{Hom}}(B, C) \cong B^{*} \otimes C$ for $B$ compact and $C$ arbitrary. In particular, taking $C=R$, we see that the object $B^{*}$ (which we defined as $\underline{\operatorname{Hom}}_{\mathrm{gm}}(B, R)$ in $\left.D M_{\mathrm{gm}}(k ; R)\right)$ is isomorphic to $\underline{\operatorname{Hom}}(B, R)$ in $D M(k ; R)$.

\section{A Künneth spectral sequence for motivic homology}

Dugger and Isaksen proved the following Künneth spectral sequence, which describes the motivic homology of the tensor product of a mixed Tate motive with any motive [18, Proposition 7.10]. Their result applies to modules over any ring spectrum in the stable homotopy category over a field $k$; the case of the EilenbergMacLane spectrum $H R$ in $S H(k)$ gives the result here, by the identification between the homotopy category of $H R$-module spectra and $D M(k ; R)$ [45, Theorem 1]. (It is also straightforward to translate Dugger and Isaksen's proof to work directly in $D M(k ; R)$.) In the case of the product of a linear scheme with any scheme over a field, this spectral sequence was constructed by Joshua [29]. 
Theorem 6.1. Let $k$ be a field. Let $R$ be a commutative ring. Let $X$ be a mixed Tate motive in $D M(k ; R)$ and $Y$ any motive in $D M(k ; R)$. For each integer $j$, there is a convergent spectral sequence

$$
E_{2}^{p q}=\operatorname{Tor}_{-p,-q, j}^{H_{*}(k, R(*))}\left(H_{*}(X, R(*)), H_{*}(Y, R(*))\right) \Rightarrow H_{-p-q}(X \otimes Y, R(j)) .
$$

This spectral sequence is concentrated in the left half-plane (columns $\leq 0$ ).

By the discussion after Theorem 7.2 , one can define a spectral sequence with the $E_{2}$ term above for any motives $X$ and $Y$ in $D M(k ; R)$. It does not always converge to the motivic homology of $X \otimes Y$.

We use cohomological numbering, which means that the differential $d_{r}$ has bidegree $(r, 1-r)$ for all $r$.

For bigraded modules $M$ and $N$ over a bigraded $\operatorname{ring} S$, $\operatorname{Tor}_{a, i, j}^{S}(M, N)$ denotes the $(i, j)$ th bigraded piece of $\operatorname{Tor}_{a}^{S}(M, N)$. For this purpose, the group $H_{i}^{M}(X, R(j))$ has bigrading $(i, j)$.

Here $H_{i}(k, R(j)) \cong H^{-i}(k, R(-j))$, and so the ring $H_{*}(k, R(*))$ is better known as the motivic cohomology ring of $k$ with coefficients in $R$. For example, $H_{-1}(k, \mathbf{Z}(-1))$ is isomorphic to $k^{*}$. More generally, $\oplus_{j \geq 0} H_{-j}(k, \mathbf{Z}(-j))$ is the Milnor $K$-theory ring, that is, the quotient of the tensor algebra generated by the abelian group $k^{*}$ by the relation $\{a, 1-a\}=0$ for each $a \in k-\{0,1\}$ [43, 52].

If $X$ and $Y$ are $k$-schemes, viewed as the motives $M^{c}(X)$ and $M^{c}(Y)$, then the spectral sequence with $R(j)$ coefficients is concentrated in columns $\leq 0$ and rows $\leq-2 j$. If we write $H_{*}(X)$ for the bigraded group $H_{*}(X, R(*))$, the $E_{2}$ term looks like:

$$
\begin{aligned}
& \begin{array}{llll}
0 & 0 & 0
\end{array} \\
& {\left[\operatorname{Tor}_{2}^{H_{*} k}\left(H_{*} X, H_{*} Y\right)\right]_{2 j, j} \quad\left[\operatorname{Tor}_{1}^{H_{*} k}\left(H_{*} X, H_{*} Y\right)\right]_{2 j, j} \quad\left[H_{*} X \otimes_{H_{*} k} H_{*} Y\right]_{2 j, j} \quad 0} \\
& {\left[\operatorname{Tor}_{2}^{H_{*} k}\left(H_{*} X, H_{*} Y\right)\right]_{2 j+1, j} \quad\left[\operatorname{Tor}_{1}^{H_{*} k}\left(H_{*} X, H_{*} Y\right)\right]_{2 j+1, j} \quad\left[H_{*} X \otimes_{H_{*} k} H_{*} Y\right]_{2 j+1, j} \quad 0}
\end{aligned}
$$

(Indeed, for a $k$-scheme $X$, the group $H_{a}(X, R(b))$ is zero unless $a \geq 2 b$, as mentioned in section 5. Since this applies to $X, Y$, and $\operatorname{Spec}(k)$, the $E_{2}$ term for the spectral sequence with $R(j)$ coefficients is concentrated in rows $\leq-2 j$.) So there

are no differentials into or out of the upper right group, $E_{2}^{0,-2 j}$. We deduce that

$$
C H_{*}\left(X \times_{k} Y ; R\right) \cong C H_{*}(X ; R) \otimes_{R} C H_{*}(Y ; R)
$$

if $X$ is a $k$-scheme with $M^{c}(X)$ a mixed Tate motive in $D M(k ; R)$ and $Y$ is any separated $k$-scheme of finite type. I proved this in the special case where $X$ is a linear scheme over $k$ [54], which helped to inspire Joshua's result.

\section{$7 \quad$ The motivic Künneth property}

In this section, we prove that a separated scheme $X$ of finite type over a field $k$ satisfies the motivic Künneth property if and only if the motive $M^{c}(X)$ is a mixed Tate motive. Given the machinery we have developed, the proof is short. 
The motivic Künneth property means that the spectral sequence described in Theorem 6.1 converges to the motivic homology of $X \times_{k} Y$ for every separated $k$ scheme $Y$ of finite type. (We recall that motivic homology groups are also called higher Chow groups.) There is a neater formulation of the Künneth property in the language of Bousfield localization, to be explained now.

The inclusion of mixed Tate motives $D M T(k ; R)$ into the category $D M(k ; R)$ of all motives has a right adjoint $D M(k ; R) \rightarrow D M T(k ; R)$, which we write as $X \mapsto C(X)$. It associates to any motive a mixed Tate motive with the same motivic homology groups. For $X$ a compact object (a geometric motive), $C(X)$ need not be a compact object. So this construction shows the convenience of "big" categories of motives. The construction is a general application of Bousfield localization, as developed by Neeman for triangulated categories.

Namely, let $\mathcal{T}$ be a triangulated category with arbitrary direct sums. Let $\mathcal{P}$ be a set of compact objects in $\mathcal{T}$. Recall from section 5 that a localizing subcategory of $\mathcal{T}$ means a full triangulated subcategory which is closed under arbitrary direct sums. Let $\mathcal{S}$ be the smallest localizing category that contains $\mathcal{P}$. Then the inclusion $\mathcal{S} \rightarrow \mathcal{T}$ has a right adjoint $C: \mathcal{T} \rightarrow \mathcal{S}$ known as colocalization with respect to $\mathcal{P}$ 41, Theorem 4.1]. By adjointness, there is a canonical morphism $C(X) \rightarrow X$, and this morphism induces a bijection $\operatorname{Hom}(P[j], C(X)) \rightarrow \operatorname{Hom}(P[j], X)$ for all objects $P$ in $\mathcal{P}$ and all integers $j$. (The localization of an object $X$ with respect to $\mathcal{P}$ means a cone $X / C(X)$, which in this case is defined up to a unique isomorphism.)

The functor $D M(k ; R) \rightarrow D M T(k ; R), X \mapsto C(X)$, mentioned above is the colocalization with respect to the compact objects $R(j)$ for $j \in \mathbf{Z}$. The construction implies that $C(X)$ is a mixed Tate motive with a morphism $C(X) \rightarrow X$ that induces isomorphisms on motivic homology groups. (That is, $\operatorname{Hom}(R(a)[b]$, $C(X)) \rightarrow \operatorname{Hom}(R(a)[b], X)$ is an isomorphism for all integers $a$ and $b$.) Moreover, $C(X)$ is determined up to a unique isomorphism by this property.

As in any triangulated category with arbitrary direct sums, the homotopy colimit $X_{\infty}=\operatorname{hocolim}\left(X_{0} \rightarrow X_{1} \rightarrow \cdots\right)$ is defined as a cone of the morphism

$$
1-s: \oplus_{i \geq 0} X_{i} \rightarrow \oplus_{i \geq 0} X_{i},
$$

where $s$ is the given map from each $X_{i}$ to $X_{i+1}[9]$.

Here is an explicit construction of the colocalization $C(X)$, modeled on Dugger and Isaksen's analogous construction in the stable homotopy category over $k$ [18, Proposition 7.3]. (They were imitating the usual construction of a cellular approximation to any topological space.) Choose a set of generators for all the motivic homology groups $H_{b}(X, R(a))$ with $a, b \in \mathbf{Z}$. Let $C_{0}$ be a direct sum of one motive $R(a)[b]$ for each generator; so we have a morphism $C_{0} \rightarrow X$ that induces a surjection on motivic homology groups. Next, choose a set of generators for the kernel of $H_{*}\left(C_{0}, R(*)\right) \rightarrow H_{*}(X, R(*))$, let $S_{1}$ be the corresponding direct sum of motives $R(a)[b]$, and let $C_{1}$ be a cone of the morphism $S_{1} \rightarrow C_{0}$. Then we have a morphism $C_{0} \rightarrow C_{1}$, and we can choose an extension of the morphism $C_{0} \rightarrow X$ to $C_{1} \rightarrow X$. Repeating the process, we get a sequence of mixed Tate motives

$$
C_{0} \rightarrow C_{1} \rightarrow \cdots
$$

with a compatible sequence of morphisms $C_{i} \rightarrow X$. These extend to a morphism from the homotopy colimit, $\operatorname{hocolim}_{j} C_{j} \rightarrow X$. This homotopy colimit is 
a mixed Tate motive, and the morphism induces an isomorphism on motivic homology groups. So the colocalization $C(X)$ is isomorphic to hocolim ${ }_{j} C_{j}$.

By Corollary 5.3, any mixed Tate motive with zero motivic homology groups is zero. This is not true for motives in general. In fact, for any motive $X$, the cone of $C(X) \rightarrow X$ has motivic homology groups equal to zero, and it is zero if and only if $X$ is a mixed Tate motive.

Lemma 7.1. The colocalization functor $X \mapsto C(X)$ from $D M(k ; R)$ to $D M T(k ; R)$ preserves arbitrary direct sums and arbitrary products.

Proof. Because the category $D M T(k ; R)$ is compactly generated, it has arbitrary products [42, Proposition 8.4.6]. (Beware that the inclusion $D M T(k ; R) \rightarrow D M(k ; R)$ preserves arbitrary direct sums, but need not preserve arbitrary products.) Because the functor $X \mapsto C(X)$ from $D M(k ; R)$ to $D M T(k ; R)$ is a right adjoint, it preserves arbitrary products. Because the functor $X \mapsto C(X)$ is colocalization with respect to a set of compact objects in $D M(k ; R)$ (namely $R(j)$ for integers $j$ ), it also preserves arbitrary direct sums [41, Theorem 5.1].

For any motives $X$ and $Y$ in $D M(k ; R)$, there is a canonical morphism

$$
C(X) \otimes C(Y) \rightarrow C(X \otimes Y),
$$

generally not an isomorphism. Indeed, tensoring the morphisms $C(X) \rightarrow X$ and $C(Y) \rightarrow Y$ gives a morphism $C(X) \otimes C(Y) \rightarrow X \otimes Y$. Since $C(X) \otimes C(Y)$ is a mixed Tate motive, this morphism factors uniquely through $C(X \otimes Y)$, as we want.

Theorem 7.2. Let $k$ be a field. Let $R$ be a commutative ring. Let $X$ be an object of the category $D M(k ; R)$ of motives (for example, $X$ could be the motive $M^{c}(W)$ for a separated $k$-scheme $W$ of finite type, if the exponential characteristic of $k$ is invertible in $R$ ). The following are equivalent.

(1) $X$ is a mixed Tate motive.

(2) $X$ satisfies the motivic Künneth property, meaning that the morphism

$$
C(X) \otimes C(M(Y)) \rightarrow C(X \otimes M(Y))
$$

of mixed Tate motives is an isomorphism for every smooth projective variety $Y$ over $k$.

(3) X satisfies the apparently stronger property that

$$
C(X) \otimes C(Y) \rightarrow C(X \otimes Y)
$$

is an isomorphism for every motive $Y$ in $D M(k ; R)$.

If $X$ belongs to the subcategory $D M_{g m}(k ; R)$ of geometric motives, for example if $X=M^{c}(B)$ for some separated $k$-scheme $B$ of finite type, then (1)-(3) are also equivalent to:

(4) $X$ is a "small" mixed Tate motive, meaning that $X$ belongs to the smallest thick subcategory of $D M(k ; R)$ that contains $R(j)$ for all integers $j$.

Let us explain why properties (2) and (3) deserve to be called Künneth properties of $X$. Since $C(X) \otimes C(Y)$ and $C(X \otimes Y)$ are both mixed Tate motives, the morphism $C(X) \otimes C(Y) \rightarrow C(X \otimes Y)$ is an isomorphism if and only if it induces an isomorphism 
on motivic homology groups, by Corollary 5.3. The motivic homology groups of $C(X \otimes Y)$ are simply the motivic homology groups of $X \otimes Y$. The motivic homology groups of $C(X) \otimes C(Y)$ are the "output" of the spectral sequence of Theorem 6.1 . with $E_{2}$ term

$\operatorname{Tor}_{*}^{H_{*}(k, *)}\left(H_{*}(C(X), R(*)), H_{*}(C(Y), R(*))\right)=\operatorname{Tor}_{*}^{H_{*}(k, *)}\left(H_{*}(X, R(*)), H_{*}(Y, R(*))\right)$.

So property (3) is saying that this Künneth spectral sequence converges to the motivic homology of $X \otimes Y$.

Proof. The Künneth property (2) is preserved under arbitrary direct sums of motives $X$, since the tensor product $\otimes$ and the functor $X \mapsto C(X)$ (by Lemma 7.1) preserve arbitrary direct sums. Also, if it holds for two of the three motives in a distinguished triangle, then it holds for the third. Finally, the motives $R(j)$ have the Künneth property. It follows that every mixed Tate motive in $D M(k ; R)$ has the Künneth property. That is, (1) implies (2).

Next, let $X$ be a motive in $D M(k ; R)$ with the Künneth property (2) with respect to smooth projective varieties over $k$. The statement that the morphism

$$
C(X) \otimes C(Y) \rightarrow C(X \otimes Y)
$$

is an isomorphism is preserved under arbitrary direct sums of motives $Y$. Also, if it holds for two motives $Y$ in a distinguished triangle, then it holds for the third. By Lemma 5.4, $X$ satisfies the Künneth property (3) with respect to all motives $Y$.

We now show that (3) implies (1). As above, the "cellular approximation" $C(X)$ is the unique mixed Tate motive with a morphism $C(X) \rightarrow X$ that induces an isomorphism on motivic homology groups. Since $C(X)$ is a mixed Tate motive, it has the Künneth property. Let $X_{2}$ be a cone of the morphism $C(X) \rightarrow X$. It suffices to show that $X_{2}=0$.

The motivic homology groups of $X_{2}$ are equal to zero. Also, $X_{2}$ satisfies the Künneth property. So the motivic homology of $X_{2} \otimes Y$ is zero for every motive $Y$ in $D M(k ; R)$. In particular, for all smooth projective varieties $Y$ over $k$ and all integers $a$ and $b$, the motivic homology group $\operatorname{Hom}\left(R, X_{2} \otimes(M(Y)(a)[b])^{*}\right)$ is zero. By Lemma 5.5, it follows that $\operatorname{Hom}\left(M(Y)(a)[b], X_{2}\right)=0$ for all smooth projective varieties $Y$ over $k$ and all integers $a$ and $b$. By Lemma 5.4, it follows that $X_{2}=0$. We have shown that (3) implies (1).

Finally, if $X$ belongs to the subcategory $D M_{\mathrm{gm}}(k ; R)$ of geometric motives, then we showed after Lemma 5.4 that (1) and (4) are equivalent.

The following consequence is not surprising, but it seems worth mentioning. Dugger and Isaksen mentioned that it is not immediately clear how to show that a given object in the stable homotopy category, $S H(k)$, for example an elliptic curve over $k$, is not cellular [18, section 1.2]. The functor $S H(k) \rightarrow D M(k ; R)$ takes cellular objects to mixed Tate motives. The following result describes which smooth projective varieties have motives which are mixed Tate motives. As a very special case, we see that elliptic curves are not mixed Tate motives (for any nonzero coefficient ring), and so elliptic curves are not cellular in $S H(k)$.

Corollary 7.3. Let $X$ be a smooth proper scheme over a field $k$. Let $R$ be a commutative ring such that the exponential characteristic of $k$ is invertible in $R$. 
If the motive $M(X)$ in $D M(k ; R)$ is a mixed Tate motive, then the Chow motive of $X$ with coefficients in $R$ is a summand of a finite direct sum of Tate motives $R(a)[2 a]$. So, for example, $C H_{*}(X) \otimes_{\mathbf{z}} R \rightarrow H_{*}\left(X_{\mathbf{C}}, R\right)$ is an isomorphism if there is an embedding $k \hookrightarrow \mathbf{C}$. In particular, $H_{*}\left(X_{\mathbf{C}}, R\right)$ is concentrated in even degrees.

Proof. By Theorem [7.2, $X$ satisfies the Künneth property for motivic homology groups with coefficients in $R$. By the discussion of the Künneth spectral sequence after Theorem 6.1, it follows that $X$ has the Chow Künneth property: the homomorphism

$$
C H_{*}(X ; R) \otimes_{R} C H_{*}(Y ; R) \rightarrow C H_{*}\left(X \times_{k} Y ; R\right)
$$

is an isomorphism for every separated $k$-scheme $Y$ of finite type. By Theorem 4.1, the Chow motive of $X$ with coefficients in $R$ is a summand of a finite direct sum of Tate motives. The theorem includes several consequences of that property, for example that $C H_{*}(X ; R) \rightarrow H_{*}\left(X_{\mathbf{C}}, R\right)$ is an isomorphism if there is an embedding $k \hookrightarrow \mathbf{C}$.

\section{The motive of a quotient stack}

Edidin and Graham defined the motivic homology of a quotient stack [19, sections 2.7 and 5.3]. In this section, we define the compactly supported motive of a quotient stack, in such a way that we recover the same motivic homology groups. One benefit of defining the motive of a quotient stack is that it makes sense to ask whether a given stack, such as $B G$ for an affine group scheme $G$, is mixed Tate, meaning that the motive $M^{c}(B G)$ is mixed Tate.

The motive $M(B G)$ (not compactly supported) in $D M(k ; R)$ was already defined, in effect, by Morel and Voevodsky [39, section 4.2]. Its motivic cohomology is the motivic cohomology of $B G$. We need to define $M^{c}(B G)$ because that is the motive relevant to the motivic homology of $B G \times X$ for separated schemes $X$ of finite type over $k$. To see the difference between the two motives, write $G_{m}$ for the multiplicative group over $k$. Then $M\left(B G_{m}\right)$ is the homotopy colimit of the motives $M\left(\mathbf{P}^{j}\right)$, and so $M\left(B G_{m}\right)$ is isomorphic to $\oplus_{j \geq 0} \mathbf{Z}(j)[2 j]$ in $D M(k ; \mathbf{Z})$. By contrast, $M^{c}\left(B G_{m}\right)$ is the homotopy limit of the motives $M\left(\mathbf{P}^{j-1}\right)(-j)[-2 j]$ by the definition below, and so $M^{c}\left(B G_{m}\right)$ is isomorphic to $\prod_{j<-1} \mathbf{Z}(j)[2 j]$ in $D M(k ; \mathbf{Z})$. This product is isomorphic to the direct sum $\oplus_{j \leq-1} \mathbf{Z}(j)[2 j]$ by Lemma 8.7, from which we see that $M^{c}\left(B G_{m}\right)$ is mixed Tate.

Another possible name for the mixed Tate property of $B G$ would be the motivic Künneth property. Indeed, by Theorem [7.2, $B G$ is mixed Tate if and only if $B G$ has the motivic Künneth property in the sense that the Künneth spectral sequence

$$
E_{2}^{p q}=\operatorname{Tor}_{-p,-q, j}^{H_{*}(k, *)}\left(H_{*}(B G, R(*)), H_{*}(Y, R(*))\right) \Rightarrow H_{-p-q}\left(B G \times_{k} Y, R(j)\right)
$$

converges to the groups on the right for every separated $k$-scheme $Y$ of finite type.

Before defining the compactly supported motive of a quotient stack, we recall the definition of homotopy limits. Let

$$
\cdots \rightarrow X_{2} \rightarrow X_{1}
$$

be a sequence of morphisms in the category $D M(k ; R)$ of motives. Since $D M(k ; R)$ is compactly generated, arbitrary products exist in $D M(k ; R)$ [42, Proposition 
8.4.6]. Dualizing Bökstedt and Neeman's definition of homotopy colimits, the homotopy limit $\operatorname{holim}_{j} X_{j}$ in $D M(k ; R)$ is defined as the fiber of the morphism $f: \prod X_{j} \rightarrow \prod X_{j}$ given by the identity minus the shift map [9]. (In other words, the homotopy limit is cone $(f)[-1]$; so it is well-defined up to isomorphism, but not necessarily up to a unique isomorphism.)

Define a quotient stack over a field $k$ to be an algebraic stack over $k$ which is the quotient stack of some quasi-projective scheme $Y$ over $k$ by an action of an affine group scheme $G$ of finite type over $k$ such that there is a $G$-equivariant ample line bundle on $Y$. (A short introduction to quotient stacks is [49, Tag 04UV]. It would be more natural to allow quotients of algebraic spaces by affine group schemes, but this definition of quotient stacks is sufficient for our applications.) For $Y$ quasi-projective over $k$, the assumption that there is a $G$-equivariant ample line bundle is automatic when $G$ is finite, or when $G$ is smooth over $k$ and $Y$ is normal, by Sumihiro's equivariant completion theorem [50] and [40, Corollary 1.6]. For example, the stack $B G$ means the quotient stack $\operatorname{Spec}(k) / G$.

The dimension of a locally Noetherian stack in defined in such a way that a quotient stack $X=A / G$ has dimension $\operatorname{dim}(A)-\operatorname{dim}(G)$ [49, Tag 0AFL]. For example, $B G$ is a smooth stack of dimension $-\operatorname{dim}(G)$ over $k$.

We can now define the compactly supported motive of a quotient stack, with coefficients in a given commutative ring $R$. Let $k$ be a field. Let $R$ be a commutative ring in which the exponential characteristic of $k$ is invertible. Let $X$ be a quotient stack over $k$. Let $\cdots \rightarrow V_{2} \rightarrow V_{1}$ be a sequence of surjections of vector bundles over $X$. Write $n_{i}$ for the rank of the bundle $V_{i}$. Think of the total space of $V_{i}$ as a stack over $k$. For each $i$, let $S_{i}$ be a closed substack of $V_{i}$ such that $V_{i}-S_{i}$ is a separated scheme and $S_{i+1}$ is contained in the inverse image of $S_{i}$ under the morphism $f_{i}: V_{i+1} \rightarrow V_{i}$ for all $i$. Assume that the codimension of $S_{i}$ in $V_{i}$ goes to infinity with $i$. (Such vector bundles $V_{i}$ and closed subsets $S_{i}$ exist because $X$ is a quotient stack. In more detail, if we write $X$ as a quotient stack $Y / G$, then we can use bundles $V_{i}$ which are given by suitable representations $V$ of $G$. Take $V_{i}-S_{i}$ to be of the form $(Y \times(V-S)) / G$ with $(V-S) / G$ a quasi-projective scheme [53, Remark 1.4]. Then $(Y \times(V-S)) / G$ is also a quasi-projective scheme by [40, Proposition 7.1], using that $Y$ has a $G$-equivariant ample line bundle.)

Define the motive $M^{c}(X)$ in $D M(k ; R)$ to be the homotopy limit of the sequence:

$$
\cdots \rightarrow M^{c}\left(V_{2}-S_{2}\right)\left(-n_{2}\right)\left[-2 n_{2}\right] \rightarrow M^{c}\left(V_{1}-S_{1}\right)\left(-n_{1}\right)\left[-2 n_{1}\right] .
$$

The morphisms here are the composition

$$
\begin{aligned}
M^{c}\left(V_{i+1}-S_{i+1}\right)\left(-n_{i+1}\right)\left[-2 n_{i+1}\right] & \rightarrow M^{c}\left(V_{i+1}-f_{i}^{-1}\left(S_{i}\right)\right)\left(-n_{i+1}\right)\left[-2 n_{i+1}\right] \\
& \cong M^{c}\left(V_{i}-S_{i}\right)\left(-n_{i}\right)\left[-2 n_{i}\right],
\end{aligned}
$$

where the first morphism is the flat pullback associated to an open inclusion, and the isomorphism follows from homotopy invariance for affine bundles. We will show that this motive is independent of the choice of vector bundles $V_{i}$ and closed substacks $S_{i}$.

Once we check that this motive is well defined in Theorem 8.5, it will be immediate that the motivic homology of a quotient stack $X=Y / G$ given by the motive $M^{c}(X)$ agrees with the motivic homology of $X$ as defined by Edidin and Graham [19, sections 2.7 and 5.3]. Namely, any given motivic homology group $H_{a}(\cdot, R(b))$ 
of the sequence above is eventually constant. In our notation, Edidin and Graham defined $H_{a}(X, R(b))$ to be equal to

$H_{a}\left(\left(\left(Y \times\left(V_{j}-S_{j}\right)\right) / G\right)\left(-n_{j}\right)\left[-2 n_{j}\right], R(b)\right) \cong H_{a+2 n_{j}}\left(\left(Y \times\left(V_{j}-S_{j}\right)\right) / G, R\left(b+n_{j}\right)\right)$

for any $j$ sufficiently large.

Tudor Padurariu observed that for a smooth quotient stack $X$ of pure dimension $n$ over $k$, the motive $M(X)$ determines $M^{c}(X)$ in a simple way: namely, $M^{c}(X) \cong$ $M(X)^{*}(n)[2 n]$. In particular, $M^{c}(B G) \cong M(B G)^{*}(-\operatorname{dim}(G))[-2 \operatorname{dim}(G)]$, since $B G$ is a smooth stack of $\operatorname{dimension}-\operatorname{dim}(G)$ over $k$. For example, it follows that

$$
C H_{i} B G \cong C H^{-\operatorname{dim}(G)-i} B G .
$$

Padurariu's argument uses that the dual of a direct sum in $D M(k ; R)$ is a product, and so the dual of a homotopy colimit is a homotopy limit. By contrast, the dual of a product does not have a simple description in general, and so it is not clear whether $M^{c}(X)^{*}$ is isomorphic to $M(X)(-n)[-2 n]$ for a smooth quotient stack $X$ of pure dimension $n$ over $k$.

The following filtration of the category $D M(k ; R)$ is very convenient for our arguments. Namely, for an integer $j$, let $D_{j}(k ; R)$ be the smallest localizing subcategory of $D M(k ; R)$ that contains $M^{c}(X)(a)$ for all separated schemes $X$ of finite type over $k$ and all integers $a$ such that $\operatorname{dim}(X)+a \leq j$. (Another possible notation would be $d_{\leq j} D M(k ; R)$, by analogy with a notation used for effective motives 27 , proof of Corollary 1.9].) Thus we have a sequence of triangulated subcategories

$$
\cdots \subset D_{-1} \subset D_{0} \subset D_{1} \subset \cdots
$$

of $D M(k ; R)$.

For an integer $j$, let $E_{j}$ be the smallest localizing subcategory of $D M(k ; R)$ that contains $M(Y)(a)$ for all smooth projective varieties $Y$ over $k$ and all integers $a>j$. This is related to the slice filtration of motives; in that setting, $E_{j}$ would be called $D M^{\mathrm{eff}}(k ; R)(j+1)$ [27, section 1]. For a triangulated subcategory $E$ of a triangulated category $T$, the right orthogonal to $E$ is the full subcategory $E^{\perp}$ of all objects $M$ such that $\operatorname{Hom}(N, M)=0$ for every $N$ in $E$ [44]. The right orthogonal $E^{\perp}$ is always a colocalizing subcategory of $T$, meaning a triangulated subcategory that is closed under arbitrary products in $T$. In the notation of the slice filtration, $E_{j}^{\perp}$ might be called $\nu_{\leq j} D M(k ; R)$ [27, Definition 1.3].

Lemma 8.1. The subcategory $D_{j}$ of $D M(k ; R)$ is contained in the right orthogonal $E_{j}^{\perp}$.

Proof. As mentioned in section 5, for any separated scheme $Z$ of finite type over $k$, we have $H_{j}(Z, R(a))=0$ for all integers $a$ and $j$ with $a>\operatorname{dim}(Z)$. Let $Y$ be a smooth projective variety over $k$, and let $n=\operatorname{dim}(Y)$. Then we have $H_{j}(Y \times Z, R(a))=0$ for all integers $a$ and $j$ with $a>n+\operatorname{dim}(Z)$. Equivalently, $\operatorname{Hom}_{D M(k ; R)}\left(R(a)[b], M(Y) \otimes M^{c}(Z)\right)=0$ for all integers $a$ and $b$ with $a>n+\operatorname{dim}(Z)$.

As mentioned in section 5 , we have

$$
M(Y)^{*} \cong M(Y)(-n)[-2 n]
$$


So $\operatorname{Hom}_{D M(k ; R)}\left(R(a)[b], M(Y)^{*} \otimes M^{c}(Z)\right)=0$ for all integers $a$ and $b$ with $a>$ $\operatorname{dim}(Z)$. By Voevodsky and Kelly's results (see the proof of Lemma 5.5), it follows that $\operatorname{Hom}_{D M(k ; R)}\left(M(Y)(a)[b], M^{c}(Z)\right)=0$ for all integers $a$ and $b$ with $a>\operatorname{dim}(Z)$. Since the object $M(Y)(a)$ is compact in $D M(k ; R)$, it follows that $\operatorname{Hom}_{D M(k ; R)}(M(Y)(a)[b]$, $N)=0$ for all motives $N$ in the subcategory $D_{j}$ and all integers $a$ and $b$ such that $a>j$. Consequently, $D_{j}$ is contained in the right orthogonal $E_{j}^{\perp}$.

Here is a convenient formal property of the subcategories $E_{j}^{\perp}$.

Lemma 8.2. For any integer $j$, the subcategory $E_{j}^{\perp}$ of $D M(k ; R)$ is both localizing and colocalizing. That is, it is a triangulated subcategory of $D M(k ; R)$ which is closed under arbitrary direct sums and arbitrary products in $D M(k ; R)$.

Proof. Since $E_{j}$ is a triangulated subcategory of $D M(k ; R), E_{j}^{\perp}$ is a triangulated subcategory of $D M(k ; R)$. As is true for any right orthogonal, $E_{j}^{\perp}$ is closed under arbitrary products in $D M(k ; R)$. Because $E_{j}$ is generated by a set of compact objects in $D M(k ; R), E_{j}^{\perp}$ is also closed under arbitrary direct sums in $D M(k ; R)$ [41, Theorem 5.1].

Lemma 8.3. The intersection of the subcategories $E_{j}^{\perp}$ of $D M(k ; R)$ for all integers $j$ is zero. It follows that the intersection of the subcategories $D_{j}$ for all integers $j$ is zero.

Proof. If a motive $N$ belongs to $E_{j}^{\perp}$ for all integers $j$, then $\operatorname{Hom}_{D M(k ; R)}(M(Y)(a)[b]$, $N)=0$ for all smooth projective varieties $Y$ over $k$ and all integers $a$ and $b$. Since the triangulated category $D M(k ; R)$ is generated by the objects $M^{c}(Y)(a)$ for smooth projective varieties $W$ over $k$ and integers $a$ (section 5), it follows that $N=0$. Thus $\cap_{j} E_{j}^{\perp}=0$. By Lemma 8.1, it follows that $\cap_{j} D_{j}=0$.

Corollary 8.4. Let $\cdots \rightarrow X_{2} \rightarrow X_{1}$ be an inverse system in $D M(k ; R)$ such that $X_{j}$ is in the subcategory $D_{a_{j}}$ with $a_{j} \rightarrow-\infty$. Then holim $X_{j}=0$.

Proof. We will show that the homotopy limit $X=\operatorname{holim}_{j} X_{j}$ belongs to $E_{m}^{\perp}$ for every integer $m$, and hence is zero by Lemma 8.3. The homotopy limit does not change if finitely many objects are removed from the inverse system. So it suffices to show that if $X_{j}$ is in $D_{a_{j}}$ with $a_{j} \leq m$ for all $j$, then the homotopy limit $X$ is in $E_{m}^{\perp}$. This is true because $D_{m}$ is contained in $E_{m}^{\perp}$ and the triangulated subcategory $E_{m}^{\perp}$ is closed under arbitrary products in $D M(k ; R)$ (Lemma 8.2).

Theorem 8.5. The compactly supported motive in $D M(k ; R)$ of a quotient stack over a field $k$ is an invariant of the stack over $k$.

Proof. Let $X$ be a quotient stack over $k$. Let $\cdots \rightarrow V_{2} \rightarrow V_{1}$ and $\cdots \rightarrow W_{2} \rightarrow W_{1}$ be two sequences of vector bundles over $X$, viewed as stacks over $k$, with closed substacks $S_{j} \subset V_{j}$ and $T_{j} \subset W_{j}$ such that $V_{j}-S_{j}$ and $W_{j}-T_{j}$ are schemes, $S_{j+1}$ is contained in the inverse image of $S_{j}$ under $V_{j+1} \rightarrow V_{j}$ and likewise for $T_{j+1}$, and the codimensions of $S_{j} \subset V_{j}$ and $T_{j} \subset W_{j}$ go to infinity. Let $m_{j}$ be the rank of the bundle $V_{j}$ over $X$ and $n_{j}$ the rank of $W_{j}$. We want to define a canonical isomorphism from the motive $X_{V}:=\operatorname{holim}_{j} M^{c}\left(V_{j}-S_{j}\right)\left(-m_{j}\right)\left[-2 m_{j}\right]$ to $X_{W}:=\operatorname{holim}_{j} M^{c}\left(W_{j}-T_{j}\right)\left(-n_{j}\right)\left[-2 n_{j}\right]$. 
Consider the sequence of vector bundles $V_{j} \oplus W_{j}$ over $X$, viewed as stacks over $k$. (These stacks are the fiber products $V_{j} \times_{X} W_{j}$.) Let $Z_{j}$ be the union of $S_{j} \times_{X} W_{j}$ and $V_{j} \times_{X} T_{j}$ inside $V_{j} \oplus W_{j}$. Then we have flat morphisms of schemes from $\left(V_{j} \oplus W_{j}\right)-Z_{j}$ to $V_{j}-S_{j}$ and to $W_{j}-T_{j}$, for all $j$. So we have morphisms from $X_{V}$ and from $X_{W}$ to the homotopy limit $X_{V W}:=\operatorname{holim}_{j} M^{c}\left(\left(V_{j} \oplus W_{j}\right)-Z_{j}\right)\left(-m_{j}-n_{j}\right)\left[-2 m_{j}-2 n_{j}\right]$, as homotopy limits of flat pullback maps of compactly supported motives. It suffices to show that these morphisms are both isomorphisms in $D M(k ; R)$.

We will show that $X_{V} \rightarrow X_{V W}$ is an isomorphism; the argument would be the same for $X_{W}$. The point is that the morphism $\left(V_{j} \oplus W_{j}\right)-Z_{j} \rightarrow V_{j}-S_{j}$ is the complement of the closed subset $V_{j} \times_{X} T_{j}$ in a vector bundle (with fiber $W_{j}$ ) over the scheme $V_{j}-S_{j}$. The vector bundle (of rank $n_{j}$ ) gives an isomorphism

$$
M^{c}\left(\left(V_{j} \oplus W_{j}\right)-\left(S_{j} \times_{X} W_{j}\right)\right)\left(-m_{j}-n_{j}\right)\left[-2 m_{j}-2 n_{j}\right] \cong M^{c}\left(V_{j}-S_{j}\right)\left(-m_{j}\right)\left[-2 m_{j}\right] .
$$

Removing $T_{j}$ changes this motive by an object in the subcategory $D_{-\operatorname{codim}\left(T_{j} \subset W_{j}\right)}$, by the localization triangle for compactly supported motives (section 5). Therefore, the cone of the morphism $X_{V} \rightarrow X_{V W}$ is a homotopy limit of motives in $D_{a_{j}}$ with $a_{j}$ approaching $-\infty$. By Corollary 8.4, this cone is zero. That is, $X_{V} \rightarrow X_{V W}$ is an isomorphism, as we want.

Our definition of the compactly supported motive of a quotient stack agrees with the standard definition in the special case of a quasi-projective scheme. As evidence that our definition is the right one for quotient stacks, we prove a localization triangle.

Theorem 8.6. Let $X$ be a quotient stack over a field $k$. Let $Y$ be a closed substack of $X$. Then there is a distinguished triangle

$$
M^{c}(Y) \rightarrow M^{c}(X) \rightarrow M^{c}(X-Y)
$$

in $D M(k ; R)$.

Proof. Write $X$ as the quotient stack $A / G$ for a quasi-projective scheme $A$ over $k$ and an affine group scheme $G$ of finite type over $k$ with a $G$-equivariant ample line bundle on $A$. Let $\cdots \rightarrow V_{2} \rightarrow V_{1}$ be a sequence of representations of $G$. Let $n_{i}=\operatorname{dim}\left(V_{i}\right)$. We can choose these representations so that there are closed subschemes $S_{i} \subset V_{i}$ such that $G$ acts freely on $V_{i}-S_{i}$ with quotient a quasi-projective scheme over $k$, $S_{i+1}$ is contained in the inverse image of $S_{i}$ for all $i$, and the codimension of $S_{i}$ in $V_{i}$ goes to infinity with $i$. Let $A_{Y}$ and $A_{X-Y}$ be the inverse images of $Y$ and $X-Y$ in the scheme $A$. Then the distinguished triangle we want is the homotopy limit of the distinguished triangles

$$
\left.M^{c}\left(\left(A_{Y} \times\left(V_{i}-S_{i}\right)\right) / G\right) \rightarrow M^{c}\left(\left(A \times\left(V_{i}-S_{i}\right)\right) / G\right) \rightarrow M^{c}\left(A_{X-Y} \times\left(V_{i}-S_{i}\right)\right) / G\right) .
$$

We now describe a basic example of the motive of a quotient stack, $M^{c}\left(B G_{m}\right)$.

Lemma 8.7. The compactly supported motive of $B G_{m}$ in $D M(k ; R)$ is isomorphic to $\prod_{j \leq-1} R(j)[2 j]$. This is isomorphic to the direct sum $\oplus_{j \leq-1} R(j)[2 j]$. 
Proof. By definition, using the representation of $G_{m}$ by scalars on an $n$-dimensional vector space for any given $n, M^{c}\left(B G_{m}\right)$ is the homotopy limit of the motives

$$
M^{c}\left(\mathbf{P}^{n-1}\right)(-n)[-2 n] \cong \prod_{j=-n}^{-1} R(j)[2 j],
$$

and so $M^{c}\left(B G_{m}\right)$ is isomorphic to the product $\prod_{j \leq-1} R(j)[2 j]$.

To show that the morphism from the direct sum $\oplus_{j \leq-1} R(j)[2 j]$ to the product is an isomorphism, it suffices to show that the cone $N$ of this morphism is zero. For any integer $a<0, N$ is isomorphic to the cone of the morphism $\oplus_{j \leq a} R(j)[2 j] \rightarrow$ $\prod_{j \leq a} R(j)[2 j]$, because finite direct sums are the same as finite products. Because the category $E_{a}^{\perp}$ is both localizing and colocalizing (Lemma 8.2), both $\oplus_{j \leq a} R(j)[2 j]$ and $\prod_{j \leq-1} R(j)[2 j]$ are in $E_{a}^{\perp}$. So $N$ is in $E_{a}^{\perp}$. Since this holds for all negative integers $a, N$ is zero by Lemma 8.3 , as we want.

Lemma 8.8. Let $X$ be a quotient stack over a field $k$. Then the motive $M^{c}(X)$ is in the subcategory $\left(E_{\operatorname{dim}(X)}\right)^{\perp}$. That is, for every smooth projective variety $Y$ over $k$,

$$
\operatorname{Hom}_{D M(k ; R)}\left(M(Y)(a)[b], M^{c}(X)\right)=0
$$

for all integers $a$ and $b$ with $a>\operatorname{dim}(X)$.

For a quotient stack $X$, one might ask whether $M^{c}(X)$ is always in the subcategory $D_{\operatorname{dim}(X)}$. For example, that is true for $M^{c}\left(B G_{m}\right)=\prod_{j \leq-1} \mathbf{Z}(j)[2 j]$, because that is isomorphic to $\oplus_{j \leq-1} \mathbf{Z}(j)[2 j]$ by Lemma 8.7, and that direct sum is in $D_{-1}$. It would be clear that the compactly supported motive of a quotient stack $X$ was in $D_{\operatorname{dim}(X)}$ if the categories $D_{m}$ were closed under arbitrary products in $D M(k ; R)$, but I suspect that they are not.

Proof. As mentioned in section 5, for any separated scheme $Z$ of finite type over $k$, we have $H_{j}(Z, R(a))=0$ for all integers $a$ and $j$ with $a>\operatorname{dim}(Z)$. Let $Y$ be a smooth projective variety over $k$, and let $n=\operatorname{dim}(Y)$. Then we have $H_{j}(Y \times Z, R(a))=0$ for all integers $a$ and $j$ with $a>n+\operatorname{dim}(Z)$. Equivalently, $\operatorname{Hom}_{D M(k ; R)}\left(R(a)[b], M(Y) \otimes M^{c}(Z)\right)=0$ for all integers $a$ and $b$ with $a>n+\operatorname{dim}(Z)$.

As mentioned in section 5, we have

$$
M(Y)^{*} \cong M(Y)(-n)[-2 n]
$$

So $\operatorname{Hom}_{D M(k ; R)}\left(R(a)[b], M(Y)^{*} \otimes M^{c}(Z)\right)=0$ for all integers $a$ and $b$ with $a>$ $\operatorname{dim}(Z)$. By the results of Voevodsky and Kelly (see the proof of Lemma [5.5), it follows that $\operatorname{Hom}_{D M(k ; R)}\left(M(Y)(a)[b], M^{c}(Z)\right)=0$ for all integers $a$ and $b$ with $a>\operatorname{dim}(Z)$.

Let $X$ be a quotient stack over $k$. By definition, $M^{c}(X)$ is a homotopy limit of motives $M^{c}(V-S)(-m)[-2 m]$, where $V$ is a vector bundle over $X$ (viewed as a stack over $k$ ) and $V-S$ is an open subscheme. The scheme $V-S$ has dimension $\operatorname{dim}(X)+m$. So the previous paragraph gives that

$$
\operatorname{Hom}_{D M(k ; R)}\left(M(Y)(a)[b], M^{c}(V-S)(-m)[-2 m]\right)=0
$$


for all integers $a$ and $b$ with $a>\operatorname{dim}(X)$. By definition of $M^{c}(X)$ as a homotopy limit, it follows that

$$
\operatorname{Hom}_{D M(k ; R)}\left(M(Y)(a)[b], M^{c}(X)\right)=0
$$

for all integers $a$ and $b$ with $a>\operatorname{dim}(X)$. That is, $M^{c}(X)$ is in the subcategory $\left(E_{\operatorname{dim}(X)}\right)^{\perp}$.

Lemma 8.9. Let $X$ be a motive in the subcategory $E_{m}^{\perp}$ of $D M(k ; R)$ for an integer $m$. Then the colocalization $C(X)$ with respect to Tate motives is in the subcategory $D_{m}$, and hence in $E_{m}^{\perp}$.

Proof. We use the construction of $C(X)$ from section 7 as a homotopy colimit $\operatorname{hocolim}_{j} C_{j}$. Since $X$ is in $E_{m}^{\perp}$, we have $H_{b}(X, R(a))=0$ for all integers $a$ and $b$ with $a>m$. So we can take the motive $C_{0}$ in the construction of $C(X)$ to be a direct sum of motives $R(a)[b]$ with $a \leq m$. Then $C_{0}$ is in $D_{m}$. So $H_{b}\left(C_{0}, R(a)\right)=0$ for all integers $a$ and $b$ with $a>m$. By induction, we can choose $C_{j}$ for all natural numbers $j$ to be in $D_{m}$. So $C(X)=\operatorname{hocolim}_{j} C_{j}$ is in $D_{m}$. By Lemma 8.1, $C(X)$ is also in $E_{m}^{\perp}$.

Define a motive $A$ in $D M(k ; R)$ to be mixed Tate modulo dimension $m$ if the cone of the morphism $C(A) \rightarrow A$ is in $E_{m}^{\perp}$. Also, define a quotient stack $X$ to be mixed Tate modulo codimension $r$ if $M^{c}(X)$ is mixed Tate modulo dimension $\operatorname{dim}(X)-r$.

Lemma 8.10. All mixed Tate motives and all motives in $E_{m}^{\perp}$ are mixed Tate modulo dimension $m$. Also, the motives that are mixed Tate modulo dimension $m$ form a triangulated subcategory of $D M(k ; R)$.

Proof. It is clear that a mixed Tate motive is mixed Tate modulo dimension $m$. Also, a motive in $E_{m}^{\perp}$ is mixed Tate modulo dimension $m$, by Lemma 8.9, It remains to show that for a distinguished triangle $X \rightarrow Y \rightarrow Z$ in $D M(k ; R)$ with $X$ and $Y$ mixed Tate modulo dimension $m, Z$ is also mixed Tate modulo dimension $m$. We have a morphism of distinguished triangles:

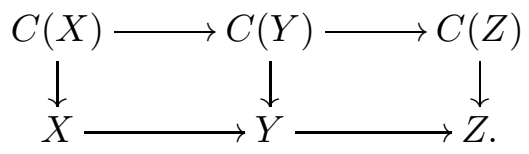

By the octahedral axiom for triangulated categories, the cone of $C(Z) \rightarrow Z$ is the cone of a morphism cone $(C(X) \rightarrow X) \rightarrow \operatorname{cone}(C(Y) \rightarrow Y)$. The latter two cones are in $E_{m}^{\perp}$, and so the cone of $C(Z) \rightarrow Z$ is also in $E_{m}^{\perp}$. That is, $Z$ is mixed Tate modulo dimension $m$.

Corollary 8.11. Let $X$ be a motive in $D M(k ; R)$ which can be approximated by mixed Tate motives in the sense that $X$ is mixed Tate modulo dimension $j$ for every integer $j$. Then $X$ is a mixed Tate motive.

Proof. The cone of $C(X) \rightarrow X$ is in $E_{j}^{\perp}$ for every integer $j$, and hence is zero by Lemma 8.3 . 
Given more geometric information on a motive $N$, the following results give better criteria for when $N$ is mixed Tate.

Lemma 8.12. Let $X$ be a separated scheme of finite type over $k$. If $X$ is mixed Tate modulo dimension -1 , then $X$ is mixed Tate.

Proof. The motive $M^{c}(X)$ is in the subcategory $E_{-1}$ of effective motives in $D M(k ; R)$, under our assumption on $R$ [33, Proposition 5.5.5]. Let $W$ be the cone of the morphism $C\left(M^{c}(X)\right) \rightarrow M^{c}(X)$. Our assumption that $X$ is mixed Tate modulo dimension -1 means that $W$ is in $E_{-1}^{\perp}$. So the morphism $M^{c}(X) \rightarrow W$ is zero. It follows that $M^{c}(X)$ is a summand of the mixed Tate motive $C\left(M^{c}(X)\right)$. So $M^{c}(X)$ is a mixed Tate motive.

There is a "finite-dimensional" criterion for when a quotient stack is mixed Tate, Corollary 8.15. Namely, a quotient stack $X=Y / G$ over $k$ is mixed Tate (meaning that $M^{c}(X)$ is mixed Tate in $\left.D M(k ; R)\right)$ if and only if the scheme $(Y \times G L(n)) / G$ is mixed Tate, for one or any faithful representation $G \hookrightarrow G L(n)$ over $k$.

Here is the main step in proving that.

Lemma 8.13. Let $X$ be a quotient stack over a field $k$. Let $E$ be a principal $G L(n)$ bundle over $X$ for some $n$, viewed as a stack over $k$. Let $r$ be an integer. Then $X$ is mixed Tate modulo codimension $r($ in $D M(k ; R))$ if and only if $E$ is mixed Tate modulo codimension $r$.

Proof. First consider the case $n=1$, so that $E$ is a principal $G_{m}$-bundle over $X$. Think of $E$ as the complement of the zero section in a line bundle over $X$. The localization triangle has the form

$$
M^{c}(X) \rightarrow M^{c}(X)(1)[2] \rightarrow M^{c}(E)
$$

in $D M(k ; R)$. Consider the morphism of distinguished triangles:

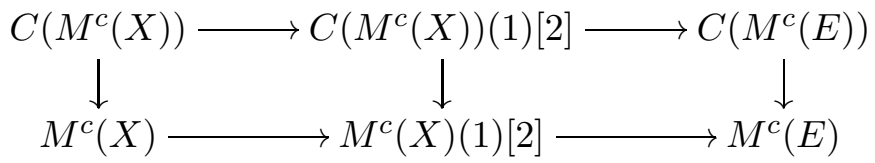

Let $W$ be the cone of $C\left(M^{c}(X)\right) \rightarrow M^{c}(X)$ and let $N$ be the cone of $C\left(M^{c}(E)\right) \rightarrow$ $M^{c}(E)$. The diagram gives a distinguished triangle $W \rightarrow W(1)[2] \rightarrow N$.

If $X$ is mixed Tate modulo codimension $r$, then $W$ is in $E_{\operatorname{dim}(X)-r}^{\perp}$. So $W(1)[2]$ is in $E_{\operatorname{dim}(X)+1-r}^{\perp}$, and hence $N$ is in $E_{\operatorname{dim}(X)+1-r}^{\perp}=E_{\operatorname{dim}(E)-r}^{\perp}$. That is, the stack $E$ is mixed Tate modulo codimension $r$, as we want.

Conversely, suppose that $E$ is mixed Tate modulo codimension $r$. That is, $N$ is in $E_{\operatorname{dim}(E)-r}^{\perp}=E_{\operatorname{dim}(X)+1-r}^{\perp}$. By Lemma 8.8, $X$ is in $E_{\operatorname{dim}(X)}^{\perp}$. By Lemma 8.9, $C(X)$ is also in $E_{\operatorname{dim}(X)}^{\perp}$, and hence $W$ is in $E_{\operatorname{dim}(X)}^{\perp}$. We want to show that $X$ is mixed Tate modulo codimension $r$, meaning that $W$ is in $E_{\operatorname{dim}(X)-r}^{\perp}$. If not, then there is a smallest integer $j$ such that $W$ is in $E_{j}^{\perp}$; we have $j>\operatorname{dim}(X)-r$ by assumption. Then $W(-1)$ is in $E_{j-1}^{\perp}$. The distinguished triangle

$$
W(-1)[-2] \rightarrow W \rightarrow N(-1)[-2]
$$


gives that $W$ is in $E_{j-1}^{\perp}$, a contradiction. Thus $X$ is mixed Tate modulo codimension $r$ if and only if the principal $G_{m}$-bundle $E$ over $X$ is mixed Tate modulo codimension $r$.

Now let $E$ be a principal $G L(n)$-bundle over a stack $X$, with $n$ arbitrary. Let $B$ be the subgroup of upper-triangular matrices in $G L(n)$ over $k$. Then $E / B$ is an iterated projective bundle over $X$, and so

$$
M^{c}(E / B) \cong \oplus_{j} M^{c}(X)\left(a_{j}\right)\left[2 a_{j}\right],
$$

where $a_{1}, \ldots, a_{n}$ ! are the dimensions of the Bruhat cells of the flag manifold $G L(n) / B$. Assume that $X$ is mixed Tate modulo codimension $r$, that is, modulo dimension $\operatorname{dim}(X)-r$. Then $M^{c}(X)(a)[2 a]$ is mixed Tate modulo dimension $\operatorname{dim}(X)-r+a$, for any integer $a$. It follows that $E / B$ is mixed Tate modulo dimension $\operatorname{dim}(X)-$ $r+\operatorname{dim}(G / B)=\operatorname{dim}(E / B)-r$. That is, $E / B$ is mixed Tate modulo codimension $r$. Conversely, if $E / B$ is mixed Tate modulo codimension $r$, then the summand $M^{c}(X)(\operatorname{dim}(G / B))[2 \operatorname{dim}(G / B)]$ of $M^{c}(E / B)$ is mixed Tate modulo dimension $\operatorname{dim}(E / B)-r=\operatorname{dim}(X)+\operatorname{dim}(G / B)-r$, and so $M^{c}(X)$ is mixed Tate modulo $\operatorname{dimension} \operatorname{dim}(X)-r$, thus modulo codimension $r$.

Next, let $U$ be the subgroup of strictly upper-triangular matrices in $G L(n)$ over $k$. Since $B / U \cong\left(G_{m}\right)^{n}$, the stack $E / U$ is a principal $\left(G_{m}\right)^{n}$-bundle over $E / B$. Applying our result on principal $G_{m}$-bundles $n$ times, we deduce that $E / U$ is mixed Tate modulo codimension $r$ if and only if $E / B$ is mixed Tate modulo codimension $r$, hence if and only if $X$ is mixed Tate modulo codimension $r$. Finally, $U$ is an extension of copies of the additive group, and so homotopy invariance gives that

$$
M^{c}(E) \cong M^{c}(E / U)(\operatorname{dim}(U))[2 \operatorname{dim}(U)] .
$$

It follows that $E$ is mixed Tate modulo codimension $r$ if and only if $X$ is mixed Tate modulo codimension $r$.

Corollary 8.14. Let $X$ be a quotient stack over a field $k$. Let $E$ be a principal $G L(n)$-bundle over $X$ for some $n$, viewed as a stack over $k$. Then $E$ is mixed Tate (in $D M(k ; R)$ ) if and only if $X$ is mixed Tate.

Proof. This follows from Lemma 8.13, since a motive is mixed Tate if and only if it is mixed Tate modulo dimension $r$ for all integers $r$ (Corollary 8.11).

Corollary 8.15. Let $Y$ be a quasi-projective scheme over a field $k$ and $G$ an affine group scheme of finite type over $k$ that acts on $Y$ such that there is a $G$-equivariant ample line bundle on $Y$. Let $G \hookrightarrow G L(n)$ be a faithful representation of $G$ over $k$. Then (the compactly supported motive of) the stack $Y / G$ over $k$ is mixed Tate if and only if the scheme $(Y \times G L(n)) / G$ over $k$ is mixed Tate.

Proof. The scheme $(Y \times G L(n)) / G$ is a principal $G L(n)$-bundle over the stack $Y / G$. So this follows from Corollary 8.14 .

For example, $B G$ is mixed Tate if and only if the scheme $G L(n) / G$ is mixed Tate, for one or any faithful representation $G \hookrightarrow G L(n)$ over $k$.

As a result, we now show that the structure of a classifying space $B G$ is determined in some ways by its properties in low codimension, namely codimension $n^{2}$ 
(roughly), where $n$ is the dimension of a faithful representation of $G$. Theorem 9.6 reduces the question of whether $B G$ is mixed Tate even further, to properties in codimension $n$ (roughly) together with properties of subgroups of $G$.

Theorem 8.16. Let $G$ be an affine group scheme over a field $k$. Suppose that $G$ has a faithful representation of dimension $n$ over $k$. If $B G$ is mixed Tate in $D M(k ; R)$ modulo codimension $n^{2}-\operatorname{dim}(G)+1$, then $B G$ is mixed Tate in $D M(k ; R)$.

Proof. We have a principal $G L(n)$-bundle $G L(n) / G \rightarrow B G$ of stacks over $k$. By Lemma 8.13, if $B G$ is mixed Tate modulo codimension $n^{2}-\operatorname{dim}(G)+1$, then the variety $G L(n) / G$ is also mixed Tate modulo codimension $n^{2}-\operatorname{dim}(G)+1$. Since $G L(n) / G$ has dimension $n^{2}-\operatorname{dim}(G)$, Lemma 8.12 gives that $G L(n) / G$ is mixed Tate. By Corollary 8.15, $B G$ is mixed Tate.

\section{$9 \quad$ The mixed Tate property for classifying spaces}

The work of Bogomolov and Saltman defines a dichotomy among all finite groups $G$ : is $B G_{\mathbf{C}}$ stably rational? (This means that the variety $V / G$ is stably rational for one, or any, faithful representation $V$ of $G$ over C.) This paper has considered several other dichotomies among finite groups $G$. Is the birational motive of $B G_{\mathbf{C}}$ trivial? Does $B G_{\mathbf{C}}$ have the weak or strong Chow Künneth property? It would be interesting to know whether these conditions are all equivalent.

Ekedahl defined another property with the same flavor, for a finite group scheme $G$ over a field $k$. Namely, when does the stack $B G$ have the class of a point in the ring $A=K_{0}\left(\operatorname{Var}_{k}\right)\left[L^{-1},\left(L^{n}-1\right)^{-1}: n \geq 1\right]$ ? Here $K_{0}\left(\operatorname{Var}_{k}\right)$ denotes the Grothendieck ring of $k$-varieties and $L$ is the class of $A^{1}$. Ekedahl showed that this property is equivalent to the statement (not mentioning stacks) that for one or any faithful representation $G \hookrightarrow G L(n)$, the variety $G L(n) / G$ is equal to $G L(n)$ in the ring $A$ [20, Proposition 3.1]. I do not know any implications between Ekedahl's property and the other properties we have mentioned, but it may be that all these properties are equivalent. In particular, Ekedahl's property fails if $G$ has nontrivial unramified $H^{2}$ [20, Theorem 5.1]; for such groups, all the properties we have mentioned fail.

In this section, we consider another dichotomy among finite groups, or more generally among affine group schemes $G$ : is $B G$ mixed Tate, meaning that the motive $M^{c}(B G)$ is mixed Tate? This property is equivalent to the motivic Künneth property formulated in the introduction to section 8 , It implies the Chow Künneth property, since it gives information about all of motivic homology, not just Chow groups. The mixed Tate property may be equivalent to all the other properties mentioned above.

We have examples of finite groups which are not mixed Tate (say over $\mathbf{C}$ ), because they do not even have the weak Chow Künneth property (Corollary 3.1). To justify the concept, we will also give examples of finite groups which are mixed Tate: the symmetric groups (Theorem 9.11), the finite general linear groups in crosscharacteristic (Theorem 9.12), and all finite subgroups of $G L(2)$ (Corollary 9.7). It is conceivable that all "naturally occurring" finite groups are mixed Tate over C. For example, Bogomolov conjectured that for every finite simple group $G$, quotient varieties $V / G$ are stably rational [8]. In that direction, Kunyavskii showed that every finite simple group has unramified $H^{2}$ equal to zero [36]. Likewise, I conjecture that 
all finite simple groups are mixed Tate. More generally, all quasisimple or almost simple groups should be mixed Tate.

In order to give examples of finite groups which are mixed Tate, we start by proving some formal properties of mixed Tate stacks. By Corollary 8.15, $B G$ is mixed Tate if and only if the variety $G L(n) / G$ is mixed Tate for a faithful representation $V$ of $G$ with $\operatorname{dim}(V)=n$. But $G L(n) / G$ may be hard to analyze because it has high dimension, namely $n^{2}$. Theorem 9.6 gives a sufficient condition for $B G$ to be mixed Tate in terms of the variety $(V-S) / G$, which has dimension only $n$, together with information on subgroups of $G$.

Throughout this section, we work in the category $D M(k ; R)$ for a field $k$ and a commutative ring $R$ in which the exponential characteristic of $k$ is invertible.

Lemma 9.1. Let $X$ be a quotient stack over a field $k$ and $Y$ a closed substack. If two of $X, Y, X-Y$ are mixed Tate, then so is the third.

Proof. This follows from the localization triangle

$$
M^{c}(Y) \rightarrow M^{c}(X) \rightarrow M^{c}(X-Y)
$$

(Theorem 8.6).

Lemma 9.2. Let $k$ be a field, and let e be the exponential characteristic of $k$. A quotient stack $X$ over a field $k$ is mixed Tate with $\mathbf{Z}[1 / e]$ coefficients (that is, in $D M(k ; \mathbf{Z}[1 / e]))$ if and only if it is mixed Tate with $\mathbf{Z}_{(p)}$ coefficients for all prime numbers $p$ that are invertible in $k$.

Proof. Write $X$ as the quotient stack $A / G$ for some affine group scheme $G$ of finite type over $k$ and some quasi-projective scheme $A$ over $k$ with a $G$-equivariant ample line bundle. Let $G \hookrightarrow G L(n)$ be a faithful representation over $k$. Then $E=$ $(A \times G L(n)) / G$ is a quasi-projective scheme over $k$, and $G L(n)$ acts on $E$ with quotient stack $E / G L(n) \cong X$. By Corollary 8.14, $M^{c}(E)$ is mixed Tate (with any coefficients) if and only if $M^{c}(X)$ is mixed Tate. So it suffices to show that $M^{c}(E)$ is mixed Tate in $D M(k ; \mathbf{Z}[1 / e])$ if and only if it is mixed Tate in $D M\left(k ; \mathbf{Z}_{(p)}\right)$ for all prime numbers $p$ that are invertible in $k$.

For a commutative ring $R, E$ is $R$-mixed Tate if and only if it has the Künneth property for the $R$-motivic homology of $E \times Y$ for all separated $k$-schemes $Y$ of finite type (Theorem 7.2). The motivic homology with $R$ coefficients of a $k$-scheme is related to motivic homology with $\mathbf{Z}$ coefficients by the universal coefficient theorem. Let $p$ be a prime number that is invertible in $k$. Since $\mathbf{Z}_{(p)}$ and $\mathbf{Z}[1 / e]$ are flat over $\mathbf{Z}$, the Künneth spectral sequence for $E \times Y$ with $\mathbf{Z}_{(p)}$ coefficients is just the localization at $p$ of the spectral sequence with $\mathbf{Z}[1 / e]$ coefficients. A homomorphism of $\mathbf{Z}[1 / e]$ modules is an isomorphism if and only if it is an isomorphism $p$-locally for all prime numbers $p$ that are invertible in $k$. Therefore, $X$ is $\mathbf{Z}[1 / e]$-mixed Tate if and only if it is $\mathbf{Z}_{(p)}$-mixed Tate for all prime numbers $p$ that are invertible in $k$.

Lemma 9.3. Let $G$ be a finite group, $p$ a prime number, and $H$ a Sylow p-subgroup of $G$. Fix a base field $k$ in which $p$ is invertible. Let $R$ be the ring $\mathbf{Z} / p$ or $\mathbf{Z}_{(p)}$. If $B H$ is R-mixed Tate, then BG is R-mixed Tate. 
Proof. Use that $B G$ is $R$-mixed Tate if and only if it has the Künneth property for $B G \times Y$ for all $k$-schemes $Y$ of finite type. Let $R$ be $\mathbf{Z} / p$ or $\mathbf{Z}_{(p)}$. Using the transfer, the Künneth spectral sequence for $B G \times Y$ is a summand with $R$ coefficients of the spectral sequence for $B H \times Y$. Therefore, if $B H$ satisfies the motivic Künneth property with $R$ coefficients, then so does $B G$.

For a representation $V$ of a finite group $G$ and $K$ a subgroup of $G, V^{K}$ means the linear subspace fixed by $K$. Following Ekedahl [20], let $V_{K}$ be the open subset of $V^{K}$ of points with stabilizer in $G$ equal to $K$, meaning that $V_{K}=V^{K}-\cup_{K \varsubsetneqq L} V^{L}$.

Lemma 9.4. Let $s$ be a natural number. Let $V$ be a faithful representation of a finite group $G$ over a field $k$. For each subgroup $K$ of $G$ that occurs as the stabilizer of a point in $V$, assume that the stack $V_{K} / N_{G}(K)$ is mixed Tate in $D M(k ; R)$ modulo codimension $s-\operatorname{codim}\left(V^{K} \subset V\right)$. Then $B G$ is mixed Tate modulo codimension s.

Proof. The stack $V / G$ is a vector bundle over $B G$. So if we can show that the stack $V / G$ is mixed Tate modulo codimension $s$, then $B G$ is mixed Tate modulo codimension $s$, as we want.

The stack $V / G$ is the disjoint union of the locally closed substacks $V_{K} / N_{G}(K)$ for all conjugacy classes of stabilizer subgroups $K$ of $G$. By assumption, each substack $V_{K} / N_{G}(K)$ is mixed Tate modulo codimension $s-\operatorname{codim}\left(V^{K} \subset V\right)$, that is, modulo dimension $\operatorname{dim}(V)-\operatorname{dim}(G)-s$. The motives that are mixed Tate modulo dimension $\operatorname{dim}(V)-s$ form a triangulated category (Lemma 8.10). By the localization triangle for stacks (Theorem 8.6), the stack $V / G$ is mixed Tate modulo dimension $\operatorname{dim}(V)-s$, that is, modulo codimension $s$.

A next step is to express the assumptions on smaller groups in terms of classifying spaces, as follows. This step may not be needed in some examples, but it leads to a neat statement, Theorem 9.6. (We will apply Lemma 9.5 to the subgroups $H=N_{G}(K)$ acting on $V^{K}$ in Lemma 9.4, typically not faithfully.)

Lemma 9.5. Let $s$ be a natural number. Let $V$ be a representation of a finite group $H$ over a field $k$, not necessarily faithful. Let $K_{1}=\operatorname{ker}(H \rightarrow G L(V))$. Consider all chains $K_{1} \varsubsetneqq K_{2} \varsubsetneqq \cdots \varsubsetneqq K_{r} \subset H, r \geq 1$, such that if we define $N_{i}=\cap_{j \leq i} N_{H}\left(K_{j}\right) \subset H$, then $K_{i+1}$ is the stabilizer of a point for $N_{i}$ acting on $V^{K_{i}}$. For every such chain, assume that $B N_{r}$ is mixed Tate in $D M(k ; R)$ modulo codimension s. (In particular, for $r=1$, we are assuming that $B H$ is mixed Tate modulo codimension s.) Then the stack $V_{K_{1}} / H$ is mixed Tate modulo codimension $s$.

Proof. By our assumption (with $r=1$ ), the stack $B H$ is mixed Tate modulo codimension $s$. So the stack $V / H$ (a vector bundle over $B H$ ) is mixed Tate modulo codimension $s$. The difference $V / H-V_{K_{1}} / H$ is the disjoint union of the locally closed substacks $\left(\coprod_{g \in H / N_{H}\left(K_{2}\right)} V_{g K_{2} g^{-1}}\right) / H$ for conjugacy classes of stabilizer subgroups $K_{2}$ for $H$ acting on $V$ with $K_{1} \varsubsetneqq K_{2}$. That quotient is isomorphic to the stack $V_{K_{2}} / N_{H}\left(K_{2}\right)$. By our assumption (with $r=2$ ), $B N_{H}\left(K_{2}\right)=B N_{2}$ is mixed Tate modulo codimension $s$, and so the stack $V^{K_{2}} / N_{H}\left(K_{2}\right)$ (a vector bundle over $\left.B N_{2}\right)$ is also mixed Tate modulo codimension $s$. The stack we want is the open 
substack $V_{K_{2}} / N_{H}\left(K_{2}\right)$ of $V^{K_{2}} / N_{H}\left(K_{2}\right)$. The complement is the disjoint union of the locally closed substacks

$$
\left(\coprod_{g \in N_{2} / N_{3}} V_{g K_{3} g^{-1}}\right) / N_{2} \cong V_{K_{3}} / N_{3},
$$

where $K_{3}$ runs over all stabilizer subgroups for $N_{2}$ acting on $V^{K_{2}}$ with $K_{1} \varsubsetneqq K_{2} \varsubsetneqq$ $K_{3}$, and $N_{N_{2}}\left(K_{3}\right)=\cap_{j \leq 3} N_{H}\left(K_{j}\right)=N_{3}$. Since $H$ is finite, the process stops after finitely many steps and gives the statement of the lemma.

Combining the previous two lemmas gives the following result. Theorem 9.6 shows that $B G$ is mixed Tate if the variety $V_{1} / G$ is mixed Tate and $B H$ is mixed Tate for certain proper subgroups $H$ of $G$. (As in the notation above, $V_{1}$ denotes the open subset of $V$ where $G$ acts freely.) Theorem 9.6 was suggested by a similar statement by Ekedahl about his invariant of $B G$ in the Grothendieck ring of varieties [20. Theorem 3.4], but I do not see a direct implication between the two results.

Theorem 9.6. Let $V$ be a faithful representation of a finite group $G$ over a field $k$. Consider all chains $1=K_{0} \varsubsetneqq K_{1} \varsubsetneqq \cdots \varsubsetneqq K_{r} \subset G, r \geq 1$, such that if we define $N_{i}=\cap_{j \leq i} N_{G}\left(K_{j}\right) \subset G, K_{i+1}$ is a stabilizer subgroup for $N_{i}$ acting on $V^{K_{i}}$. Suppose that the variety $V_{1} / G$ is mixed Tate in $D M(k ; R)$ and that the stack $B N_{r}$ is mixed Tate for all such chains with $N_{r} \neq G$. Then $B G$ is mixed Tate.

Proof. We show by induction on $s$ that $B G$ is mixed Tate modulo codimension $s$ for every natural number $s$. That will imply that $B G$ is mixed Tate by Corollary 8.11 (or by the stronger Theorem 8.16). Clearly $B G$ is mixed Tate modulo codimension 0 . Suppose that $B G$ is mixed Tate modulo codimension $s$. To show that $B G$ is mixed Tate modulo codimension $s+1$, we use Lemma 9.4. So it suffices to show that for each stabilizer subgroup $K_{1}$ of $G$ acting on $V$, the stack $V_{K_{1}} / N_{G}\left(K_{1}\right)$ is mixed Tate modulo codimension $s+1-\operatorname{codim}\left(V^{K_{1}} \subset V\right)$. For $K_{1}=1$, this is true, because we assume that the variety $V_{1} / G$ is mixed Tate. It remains to consider a stabilizer subgroup $K_{1} \neq 1$. We apply Lemma 9.5 to the vector space $V^{K_{1}}$ with its action of $N_{G}\left(K_{1}\right)$. If $N_{G}\left(K_{1}\right) \neq G$, then Lemma 9.5 and our assumptions imply that the stack $V_{K_{1}} / N_{G}\left(K_{1}\right)$ is mixed Tate. Finally, if $K_{1} \neq 1$ and $N_{G}\left(K_{1}\right)=G$, then Lemma 9.5, our assumptions, and the inductive hypothesis that $B G$ is mixed Tate modulo codimension $s$ imply that the stack $V_{K_{1}} / N_{G}\left(K_{1}\right)$ is mixed Tate modulo codimension $s$. This implies that $V_{K_{1}} / N_{G}\left(K_{1}\right)$ is mixed Tate modulo codimension $s+1-\operatorname{codim}\left(V^{K_{1}} \subset V\right)$ (as we want), because $\operatorname{codim}\left(V^{K_{1}} \subset V\right)>0$, since $K_{1} \neq 1$ and $G$ acts faithfully on $V$. The induction is complete. So $B G$ is mixed Tate.

We now use Theorem 9.6 to give examples of finite groups which are mixed Tate. (The assumption on the field $k$ in Corollary 9.7 could be weakened.) For example, Corollary 9.7 gives that the dihedral groups, generalized quaternion groups, modular 2-groups, and semidihedral groups [1, section 23.4] are mixed Tate.

Corollary 9.7. Let $k$ be a field that contains $\overline{\mathbf{Q}}$. Let $G$ be a finite subgroup of $G L(2)$ over $k$. Then $B G$ is mixed Tate in $D M(k ; \mathbf{Z})$.

Proof. Use induction on the order of $G$. Let $V$ be the given 2-dimensional faithful representation of $G$. Since $B H$ is mixed Tate for all proper subgroups $H$ of $G$, Theorem 9.6 shows that $B G$ is mixed Tate if the variety $V_{1} / G$ is mixed Tate. 
The group $G$ acts on the projective space $\mathbf{P}^{1}$ of lines in $V_{1}$. The coarse quotient $\mathbf{P}^{1} / G$ is a normal projective curve over $k$, and so it is smooth over $k$. It is unirational over $k$, and hence isomorphic to $\mathbf{P}^{1}$ over $k$.

It is convenient to observe that the representation $V$ of $G$ can be defined over $\overline{\mathbf{Q}}$. Let $S$ be the closed subset of $\mathbf{P}^{1}$ where $G$ does not act freely; then $\left(\mathbf{P}^{1}-S\right) / G$ is isomorphic to $\mathbf{P}^{1}-T$ for some closed subset $T$. Since $S$ and $T$ are defined over $\overline{\mathbf{Q}}, T$ is a finite union of copies of $\operatorname{Spec}(k)$. So $\mathbf{P}^{1}-T$ is a linear scheme over $k$ (as defined in section 5). An open subset of $V_{1} / G$ is a principal $G_{m}$-bundle over $\mathbf{P}^{1}-T$, and hence is a linear scheme over $k$. The complement of this open subset is the union of finitely many curves of the form $G_{m} / H$ where $H$ is a finite subgroup of $G_{m}$; these are isomorphic to $G_{m}$ and hence are linear schemes over $k$. So $V_{1} / G$ is a linear scheme over $k$. Thus $V_{1} / G$ is mixed Tate, and so $B G$ is mixed Tate.

We now show that many wreath product groups are mixed Tate. It will follow that the finite general linear groups in cross-characteristic and the symmetric groups are mixed Tate (Theorems 9.11 and 9.12), since their Sylow $p$-subgroups are products of iterated wreath products of cyclic groups. This is related to Voevodsky's construction of Steenrod operations on motivic cohomology, which can be viewed as computing the motivic cohomology of the symmetric groups over any field 58 , section 6], [59.

Lemma 9.8. Let $k$ be a field of characteristic not $p$ that contains the pth roots of unity. Let $X$ be a quasi-projective linear scheme over $k$ (as defined in section 5 ). Then the cyclic product $Z^{p} X=X^{p} /(\mathbf{Z} / p)$ is a quasi-projective linear scheme over $k$.

We assume that $X$ is quasi-projective in order to ensure that the cyclic product $Z^{p} X$ is a scheme. If we worked with algebraic spaces throughout, then the assumption of quasi-projectivity would be unnecessary.

Proof. We start by showing that for any representation $V$ of $\mathbf{Z} / p$ over $k$, the quotient variety $V /(\mathbf{Z} / p)$ is a linear scheme, following [53, proof of Lemma 8.1]. We use induction on the dimension of $V$. We can assume that $\mathbf{Z} / p$ acts nontrivially on $V$. Then we can write $V=W \oplus L$, where $L$ is a nontrivial 1-dimensional representation of $\mathbf{Z} / p$. The quotient variety $V /(\mathbf{Z} / p)$ has a closed subvariety $W /(\mathbf{Z} / p)$, which is a linear scheme by induction. The open complement is a vector bundle (with fiber $W)$ over $(L-0) /(\mathbf{Z} / p) \cong A^{1}-0$. A direct calculation shows that this vector bundle is trivial. So the open complement is isomorphic to $W \times\left(A^{1}-0\right)$, which is a linear scheme. Thus $V /(\mathbf{Z} / p)$ is a linear scheme over $k$, completing the induction.

Next, let $Y$ be a closed subscheme of a scheme $X$ over $k$, and let $U=X-Y$. Then the cyclic product scheme $Z^{p} X$ is the disjoint union (as a set) of $Z^{p} Y, Z^{p} U$, and various products $Y^{a} \times U^{p-a}$ for $0 \leq a \leq p$. Suppose that $X, Y$, and $U$ are linear schemes over $k$. Then all products $Y^{a} \times U^{p-a}$ are linear schemes. As a result, if any two of $Z^{p} X, Z^{p} Y$, and $Z^{p} U$ are linear schemes, then so is the third. By the inductive definition of linear schemes, it follows that for every linear scheme $X$ over $k, Z^{p} X$ is a linear scheme over $k$.

Let $G$ be an affine group scheme of finite type over a field $k$. We say that $B G$ can be approximated by linear schemes over $k$ if, for every natural number $r$, there 
is a representation $V$ of $G$ and a closed $G$-invariant subset $S$ of codimension at least $r$ in $V$ such that $G$ acts freely on $V-S$ and $(V-S) / G$ is a linear scheme over $k$. If $B G$ can be approximated by linear schemes, then $B G$ is mixed Tate. Indeed, for each $r, V, S$ as just mentioned, the compactly supported motive of the quotient stack $S / G$ is in the subcategory $\left(E_{\operatorname{dim}(S)-\operatorname{dim}(G)}\right)^{\perp}$, by Lemma 8.8. Write $V / G$ for the quotient stack. Then it follows that the cone of the morphism

$$
M^{c}(B G) \cong M^{c}(V / G)(-\operatorname{dim}(V))[-2 \operatorname{dim}(V)] \rightarrow M^{c}(V-S) / G(-\operatorname{dim}(V))[-2 \operatorname{dim}(V)]
$$

lies in $\left(E_{\operatorname{dim}(S)-\operatorname{dim}(V)-\operatorname{dim}(G)}\right)^{\perp}$, hence in $\left(E_{-r-\operatorname{dim}(G)}\right)^{\perp}$. Since we assumed that $r$ can be arbitrarily large, Corollary 8.11 gives that $M^{c}(B G)$ is mixed Tate.

For a group $G$, the wreath product $\mathbf{Z} / p \nmid G$ means the semidirect product $\mathbf{Z} / p \ltimes G^{p}$, with $\mathbf{Z} / p$ cyclically permuting the copies of $G$.

Lemma 9.9. Let $k$ be a field of characteristic not $p$ that contains the pth roots of unity. Let $G$ be an affine group scheme over $k$ such that $B G$ can be approximated by linear schemes over $k$. Then $B(\mathbf{Z} / p \nmid G)$ can be approximated by linear schemes over $k$, and hence is mixed Tate.

Proof. Let $V$ be a representation of $G$ over $k$. Then $V^{\oplus p}$ can be viewed as a representation of $\mathbf{Z} / p \nmid G$, where $\mathbf{Z} / p$ permutes the copies of $V$. If the quotients make sense, then we have $V^{\oplus p} /(\mathbf{Z} / p \curlywedge G)=Z^{p}(V / G)$. It follows that if $B G$ can be approximated by linear schemes $Y$, then $B(\mathbf{Z} / p<G)$ is approximated by the schemes $Z^{p} Y$, which are linear schemes by Lemma 9.8 .

Corollary 9.10. Let $G$ be a group scheme over a field $k$ that satisfies one of the following assumptions. Then $B G$ is mixed Tate in $D M(k ; \mathbf{Z})$.

1. $G$ is the multiplicative group $G_{m}$.

2. $G$ is a finite abelian group of exponent e viewed as an algebraic group over $k$, $e$ is invertible in $k$, and $k$ contains the eth roots of unity.

3. $G$ is an iterated wreath product $\mathbf{Z} / p \nmid \cdots\urcorner \mathbf{Z} / p \nmid G_{m}$ over $k, p$ is invertible in $k$, and $k$ contains the pth roots of unity.

4. $G$ is an iterated wreath product $\mathbf{Z} / p \nmid \cdots \curlywedge \mathbf{Z} / p \nmid A$ for a finite abelian group $A$ of exponent $e$, viewed as an algebraic group over $k$. Also, $p$ and e are invertible in $k$ and $k$ contains the pth and eth roots of unity.

Proof. In all these cases, $B G$ can be approximated by linear schemes over $k$ and hence is mixed Tate. First, $B G_{m}$ can be approximated by the schemes $\left(A^{n}-\right.$ $0) / G_{m}=\mathbf{P}^{n-1}$ over $k$ as $n$ increases. These are linear schemes. Next, when $A$ is a finite abelian group of exponent $e$ such that $e$ is invertible in $k$ and $k$ contains the eth roots of unity, then $A$ is isomorphic to a product of the group schemes $\mu_{r}$ over $k$. The classifying space $B \mu_{r}$ can be approximated by the schemes $\left(A^{n}-0\right) / \mu_{r}$ as $n$ increases, where $\mu_{r}$ acts by scalars. This scheme is the total space of the line bundle $O(r)$ minus the zero section over $\mathbf{P}^{n-1}$, and hence is a linear scheme. So $B A$ can be approximated by linear schemes, under our assumption on $k$. Finally, the statements on wreath products follow from Lemma 9.9. 
Theorem 9.11. Let $n$ be a positive integer, and let $k$ be a field of characteristic zero that contains the pth roots of unity for all primes $p$ dividing $n$. Then the symmetric group $S_{n}$ is mixed Tate over $k$ (with $\mathbf{Z}$ coefficients).

Proof. Let $p$ be a prime number. A Sylow $p$-subgroup $H$ of $G=S_{n}$ is a product of iterated wreath products $\mathbf{Z} / p \nmid \cdots \succ \mathbf{Z} / p$. By Corollary 9.10, $B H$ is mixed Tate in $D M(k ; \mathbf{Z})$, hence in $D M\left(k ; \mathbf{Z}_{(p)}\right)$ by Lemma 9.2. By Lemma 9.3, $B G$ is mixed Tate in $D M\left(k ; \mathbf{Z}_{(p)}\right)$. Since this holds for all prime numbers $p, B G$ is mixed Tate in $D M(k ; \mathbf{Z})$ by Lemma 9.2 .

Theorem 9.12. Let $n$ be a positive integer, $q$ a power of a prime number $p$, and $l$ a prime number different from $p$. Let $r$ be the order of $q$ in $(\mathbf{Z} / l)^{*}$, and let $\nu$ be the $l$-adic order of $q^{r}-1$. If $l=2$, assume that $q \equiv 1(\bmod 4)$. Let $k$ be a field of characteristic not $l$ that contains the $l^{\nu}$ roots of unity. Then the finite group $G L\left(n, \mathbf{F}_{q}\right)$ is mixed Tate in $\operatorname{DM}\left(k, \mathbf{Z}_{(l)}\right)$.

Proof. If $l$ is odd, or if $l=2$ and $q \equiv 1(\bmod 4)$, then a Sylow $l$-subgroup of $G L\left(n, \mathbf{F}_{q}\right)$ is a product of wreath products $\mathbf{Z} / l \imath \cdots \imath \mathbf{Z} / l<\mathbf{Z} / l^{\nu}$ [10, 60]. The result follows from Corollary 9.10 and Lemma 9.3 .

\section{Groups of order 32}

Let $G$ be a $p$-group of order at most $p^{4}$, for a prime number $p$. Let $e$ be the exponent of $G$. Let $k$ be a field of characteristic not $p$ which contains the eth roots of unity. Then the Chow ring of $B G$ consists of transferred Euler classes of representations [55, Theorem 11.1], and this remains true over every extension field of $k$. All representations of a subgroup of $G$ over an extension field of $k$ can be defined over $k$, and so it follows that $G$ has the weak Chow Künneth property: $C H^{*} B G \rightarrow C H^{*} B G_{E}$ is surjective for every extension field $E$ of $k$.

In this section, we show that groups of order 32 also satisfy the weak Chow Künneth property. It follows that the results after Corollary 3.1 are optimal: there are groups of order 64 , and of order $p^{5}$ for any odd prime number $p$, which do not have the weak Chow Künneth property.

Our proof of the weak Chow Künneth property for groups $G$ of order 32 uses the fact that $B G$ is stably rational for these groups, by $\mathrm{Chu}, \mathrm{Hu}$, Kang, and Prokhorov [12]. We do not know how to relate these two properties in general; as discussed in section 9, they may be equivalent.

Theorem 10.1. Let $G$ be a group of order 32. Let e be the exponent of $G$. Let $k$ be a field of characteristic not 2 which contains the eth roots of unity. Then BG over $k$ satisfies the weak Chow Künneth property.

Proof. For every proper subgroup $H$ of $G, H$ has order dividing 16, and so $B H$ over $k$ satisfies the weak Chow Künneth property, as mentioned above.

Let $V$ be a faithful representation of $G$ over $k$. Since $k$ does not have characteristic $2, V$ is a direct sum of irreducible representations, $V=\oplus_{i=1}^{c} V_{i}$. Write $P(W)$ for the space of hyperplanes in a vector space $W$, so that $P\left(W^{*}\right)$ is the space of lines in $W$. Then $G$ acts on the product of projective spaces $Y=P\left(V_{1}^{*}\right) \times \cdots \times P\left(V_{c}^{*}\right)$. The kernel of the action of $G$ on $Y$ is the center of $G$, by Schur's lemma. Let $D_{Y}$ 
be the closed subset of $Y$ where $G / Z(G)$ does not act freely. Let $D$ be the union of $\cup_{i=1}^{c} \oplus_{j \neq i} V_{j}$ with the inverse image of $D_{Y}$ in $V$. Then $D$ is a $G$-invariant finite union of linear subspaces of $V$, and $D \neq V$.

Lemma 10.2. Let $G$ be a p-group. Let $V$ be a faithful representation of $G$ over a field $k$ of characteristic not $p$. Let $Y$ be the product of projective spaces defined above, and define $D_{Y}$ and $D$ as above. Suppose that the variety $(V-D) / G$ has the weak Chow Künneth property. Also, suppose that for every subgroup $N \neq G$ that is the stabilizer of some intersection of irreducible components of $D$ (as a set), $B N$ has the weak Chow Künneth property. Then BG has the weak Chow Künneth property.

Lemma 10.2 is analogous to Theorem 9.6 on the mixed Tate property, but the argument for the weak Chow Künneth property is simpler.

Proof. (Lemma 10.2) By the localization sequence for Chow groups of quotient stacks [19, section 2.7], if a quotient stack $X$ over $k$ has the weak Chow Künneth property, then so does every open substack of $X$. Also, if a closed substack $S$ of $X$ and $X-S$ both have the weak Chow Künneth property, then so does $X$. We sometimes write CK for Chow Künneth.

We need some variants of these statements. For an integer $a$, say that a quotient stack $X$ has the weak CK property in dimension at least a if $C_{i} X \rightarrow C H_{i} X_{E}$ is surjective for all fields $E / k$ and all $i \geq a$. Also, say that $X$ has the weak CK property in codimension $b$ if $X$ has the weak CK property in dimension at least $\operatorname{dim}(X)-b$. By the localization sequence for Chow groups, if $X$ has the weak CK property in codimension $b$, then so does any open substack of the same dimension as $X$. Also, if a closed substack $S$ of $X$ and $X-S$ both have the weak CK property in dimension at least $a$, then so does $X$.

To prove the lemma, we show by induction on $b$ that $B G$ has the weak Chow Künneth property in codimension $b$ for all $b$. This is clear for $b=-1$. Suppose that $B G$ has the weak CK property in codimension $b$. To show that $B G$ has the weak CK property in codimension $b+1$, it is equivalent to show that the stack $V / G$ (a vector bundle over $B G$ ) has the weak CK property in codimension $b+1$. We are assuming that the variety $(V-D) / G$ has the weak CK property. Its complement in the stack $V / G$ is a finite disjoint union of locally closed substacks of the form $U / N$, where $U$ is an open subset of a linear subspace $W \varsubsetneqq V$ and $N$ is the stabilizer in $G$ of $W$ as a set. If $N \neq G$, then we are assuming that $B N$ has the weak CK property. So the stack $W / N$ (a vector bundle over $B N$ ) has the weak CK property, and hence the open substack $U / N$ has the weak CK property. On the other hand, if $N=G$, then $B G$ has the weak CK property in codimension $b$ by the inductive assumption, and so the stack $W / G$ and its open substack $U / G$ have the weak CK property in codimension $b$. Here $W$ has codimension $>0$ in $V$. We conclude that the stack $V / G$ has the weak CK property in codimension $b+1$, thus completing the induction. So $B G$ has the weak CK property.

We continue the proof of Theorem 10.1. Let $G$ be a group of order 32. Let $e$ be the exponent of $G$, and let $k$ be a field of characteristic not 2 that contains the eth roots of unity. If $G$ is not isomorphic to $(\mathbf{Z} / 2)^{5}$, then $G$ has a faithful complex representation $V$ of dimension 4 . (This can be checked using the free group-theory 
program GAP [23], or by the methods of Cernele-Kamgarpour-Reichstein [11, proof of Lemma 13].) The group $(\mathbf{Z} / 2)^{5}$ has the weak CK property as we want, and so we can assume that $G$ has a faithful representation of dimension 4 . The representation theory of $G$ is the "same" over $k$ as over $\mathbf{C}$, and so $G$ has a faithful representation $V$ of dimension 4 over $k$. As above, write $V=\oplus_{i=1}^{c} V_{i}$, and $Y=P\left(V_{1}^{*}\right) \times \cdots \times P\left(V_{c}^{*}\right)$.

By Lemma 10.2, $B G$ over $k$ has the weak CK property if the $k$-variety $(V-$ $D) / G$ of dimension 4 has the weak CK property. The variety $(V-D) / G$ is a principal bundle over $\left(Y-D_{Y}\right) /(G / Z(G))$, with fiber $\left(G_{m}\right)^{c} / Z(G) \cong\left(G_{m}\right)^{c}$. (The representation $V$ gives an inclusion of the center $Z(G)$ into $\left(G_{m}\right)^{c}$, which describes the scalar by which an element of the center acts on each irreducible summand $V_{i}$.) So the pullback homomorphism

$$
C H^{*}\left(Y-D_{Y}\right) /(G / Z(G)) \rightarrow C H^{*}(V-D) / G
$$

is surjective. The variety $\left(Y-D_{Y}\right) /(G / Z(G))$ has dimension $4-c$, which is at most 3. As a result, $C H^{*}(V-D) / G$ is concentrated in degrees at most $4-c$.

The group $C H^{i} B G$ is always generated by Chern classes of representations for $i \leq 2$ [53, Theorem 3.2]. All representations of $G$ over an extension field of $k$ can be defined over $k$, and so $B G$ has the weak CK property in codimension 2 (meaning that $C H^{i} B G \rightarrow C H^{i} B G_{E}$ is surjective for any $i \leq 2$ and any field extension $E$ of $k$ ). So the stack $V / G$ and hence the variety $(V-D) / G$ have the weak CK property in codimension 2. If $c \geq 2$, meaning that $V$ is reducible, then $C H^{*}(V-D) / G$ is concentrated in degrees at most 2 by the previous paragraph. So $(V-D) / G$ has the weak CK property and we are done.

There remains the case where $c=1$, that is, where $G$ has a faithful irreducible representation $V$ of dimension 4 over $k$. In this case, $C H^{*}(V-D) / G$ is concentrated in degrees at most 3, and this remains true over any extension field of $k$. We know that $(V-D) / G$ has the weak CK property in codimension 2, and we want to show that it has the weak CK property in codimension 3.

We use the fact that $B G$ is stably rational over $k$ for all groups $G$ of order 32, under our assumption on $k$, by $\mathrm{Chu}, \mathrm{Hu}$, Kang, and Prokhorov [12. This means that the variety $(V-D) / G$ is stably rational over $k$. Since $(V-D) / G$ is a principal $G_{m}$-bundle over the 3-fold $\left(Y-D_{Y}\right) /(G / Z(G))$, that 3-fold is also stably rational over $k$. It follows that $C H^{3}\left(Y-D_{Y}\right) /(G / Z(G))$ is generated by a $k$-rational point on $\left(Y-D_{Y}\right) /(G / Z(G))$, and this remains true over every extension field of $k$. So $\left(Y-D_{Y}\right) /(G / Z(G))$ has the weak CK property in codimension 3. By the surjection $C H^{3}\left(Y-D_{Y}\right) /(G / Z(G)) \rightarrow C H^{3}(V-D) / G$, which remains true over every extension field of $k,(V-D) / G$ has the weak CK property in codimension 3. By what we have said, this completes the proof that $B G$ has the weak CK property.

\section{References}

[1] M. Aschbacher. Finite group theory. Cambridge (2000). 35

[2] A. Auel, J.-L. Colliot-Thélène, and R. Parimala. Universal unramified cohomology of cubic fourfolds containing a plane. arXiv:1310.6705 11 
[3] S. Bloch. The moving lemma for higher Chow groups. J. Alg. Geom. 3 (1994), 537-568. 15

[4] S. Bloch. Lectures on algebraic cycles. 2nd ed., Cambridge (2010). 10

[5] S. Bloch and V. Srinivas. Remarks on correspondences and algebraic cycles. Amer. J. Math. 105 (1983), 1235-1253. 10

[6] F. A. Bogomolov and P. I. Katsylo. Rationality of some quotient varieties. Mat. Sb. 126 (168) (1985), 584-589. 8

[7] F. A. Bogomolov. The Brauer group of quotient spaces of linear representations. Izv. Akad. Nauk SSSR 51 (1987), 485-516, 688; translation in Math. USSR-Izv. 30 (1988), 455-485. 8

[8] F. A. Bogomolov. Stable cohomology of groups and algebraic varieties. Mat. Sb. 183 (1992), no. 5, 3-28; translation in Russian Acad. Sci. Sb. Math. 76 (1993), no. 1, 1-21. 32

[9] M. Bökstedt and A. Neeman. Homotopy limits in triangulated categories. Compos. Math. 86 (1993), 209-234. 16, 20, 24

[10] R. Carter and P. Fong. The Sylow 2-subgroups of the finite classical groups. $J$. Algebra 1 (1964), 139-151. 38

[11] S. Cernele, M. Kamgarpour, and Z. Reichstein. Maximal representation dimension for groups of order $p^{n}$. J. Group Theory 14 (2011), no. 4, 637-647. 40

[12] H. Chu, S.-J. Hu, M. Kang, and Y. G. Prokhorov. Noether's problem for groups of order 32. J. Algebra 320 (2008), 3022-3035. 8, 38, 40,

[13] H. Chu and M. Kang. Rationality of p-group actions. J. Algebra 237 (2001), 673-690. 8

[14] D.-C. Cisinski and F. Déglise. Local and stable homological algebra in Grothendieck abelian categories. Homology, Homotopy Appl. 11 (2009), 219260. 13, 14

[15] D.-C. Cisinski and F. Déglise. Triangulated categories of mixed motives. arXiv:0912.2110 15

[16] D.-C. Cisinski and F. Déglise. Integral mixed motives in equal characteristic. arXiv:1410.6359 14

[17] J.-L. Colliot-Thélène. Birational invariants, purity and the Gersten conjecture. K-theory and algebraic geometry (Santa Barbara, 1992), 1-64. Proc. Sympos. Pure Math. 58, Part 1, Amer. Math. Soc. (1995). 9

[18] D. Dugger and D. Isaksen. Motivic cell structures. Algebr. Geom. Topol. 5 (2005), 615-652. 16, 17, 18, 20, 22

[19] D. Edidin and W. Graham. Equivariant intersection theory. Inv. Math. 131 (1998), 595-634. 9, 23, 24, 39. 
[20] T. Ekedahl. A geometric invariant of a finite group. arXiv:0903.3148v1 2, 32, 34, 35

[21] A. Elmendorf, I. Kriz, M. Mandell, and J. P. May. Modern foundations for stable homotopy theory. Handbook of algebraic topology, 213-253. North-Holland (1995). 14

[22] W. Fulton. Intersection theory. Springer (1998). 3, 4

[23] The GAP Group. GAP - Groups, Algorithms, and Programming, Version 4.6.4 (2013). http://www.gap-system.org 40

[24] A. Grothendieck. Éléments de géométrie algébrique. IV. Étude locale des schémas et des morphismes de schémas. II. Publ. Math. IHES 24 (1965), 231 pp. 3

[25] A. Hoshi, M. Kang, and B. Kunyavskii. Noether's problem and unramified Brauer groups. Asian J. Math. 17 (2013), 689-713. arXiv:1202.5812 8, 9,

[26] M. Hovey. Model categories. American Mathematical Society (1999). 15

[27] A. Huber and B. Kahn. The slice filtration and mixed Tate motives. Compos. Math. 142 (2006), 907-936. 25

[28] U. Jannsen. Motivic sheaves and filtrations on Chow groups. Motives (Seattle, 1991), 245-302. Proc. Sympos. Pure Math. 55, Amer. Math. Soc. (1994). 10

[29] R. Joshua. Algebraic $K$-theory and higher Chow groups of linear varieties. Math. Proc. Cambridge Phil. Soc. 130 (2001), 37-60. 18

[30] B. Kahn. Algebraic $K$-theory, algebraic cycles and arithmetic geometry. Handbook of $K$-Theory, v. 1, 351-428. Springer (2005). 9

[31] B. Kahn and R. Sujatha. Birational motives, I: pure birational motives. arXiv:0902.4902v1 3, 5

[32] N. Karpenko and A. Merkurjev. On standard norm varieties. Ann. Sci. École Norm. Sup. 46 (2013), 175-214. 3, 7

[33] S. Kelly. Triangulated categories of motives in positive characteristic. arXiv:1305.5349 14, 15, 16, 17, 18, 30,

[34] S.-I. Kimura. Surjectivity of the cycle map for Chow motives. Motives and algebraic cycles, 157-165. Fields Inst. Commun. 56, Amer. Math. Soc. (2009). 10

[35] S. Kleiman. Motives. Algebraic geometry, Oslo 1970, 53-82. Wolters-Noordhoff (1972). 10

[36] B. Kunyavskii. The Bogomolov multiplier of finite simple groups. Cohomological and geometric approaches to rationality problems, 209-217. Birkhäuser Boston (2010). 32 
[37] M. Levine. Techniques of localization in the theory of algebraic cycles. J. Alg. Geom. 10 (2001), 299-363. 15

[38] A. Merkurjev. Unramified elements in cycle modules. J. London Math. Soc. 78 (2008), 51-64. 3, 5

[39] F. Morel and V. Voevodsky. $A^{1}$-homotopy theory of schemes. Publ. Math. IHES 90 (1999), 45-143. 1, 23

[40] D. Mumford, J. Fogarty, and F. Kirwan. Geometric invariant theory. Springer (1994). 24

[41] A. Neeman. The Grothendieck duality theorem via Bousfield's techniques and Brown representability. J. Amer. Math. Soc. 9 (1996), 205-236. 16, 17, 20, 21, 26

[42] A. Neeman. Triangulated categories. Princeton (2001). 21, 24,

[43] Yu. P. Nesterenko and A. A. Suslin. Homology of the general linear group over a local ring, and Milnor's K-theory. (Russian) Izv. Akad. Nauk SSSR Ser. Mat. 53 (1989), no. 1, 121-146. Translation in Math. USSR Izv. 34 (1990), 121-145. 19

[44] D. Orlov. Derived categories of coherent sheaves and triangulated categories of singularities. Algebra, arithmetic, and geometry: in honor of Yu. I. Manin, vol. 2, 503-531. Birkhäuser (2009). 25

[45] O. Röndigs and P. Østvær. Modules over motivic cohomology. Adv. Math. 219 (2008), 689-727. 13, 14, 16, 18

[46] M. Rost. Chow groups with coefficients. Doc. Math. 1 (1996), 319-393. 4, 7 , 10

[47] A. Scholl. Classical motives. Motives (Seattle, 1991), Proc. Sympos. Pure Math. 55, Amer. Math. Soc. (1994), 163-187. 11, 12

[48] E. Shinder. Künneth formula for motivic cohomology. MathOverflow (2010), http://mathoverflow.net/questions/6834/kunneth-formula-for-motivic-cohomology/12948\#12948 10

[49] The Stacks Project Authors. Stacks Project (2014). http://stacks.math.columbia.edu 24

[50] H. Sumihiro. Equivariant completion II. J. Math. Kyoto Univ. 15 (1975), 573605. 24

[51] A. Suslin. Algebraic $K$-theory of fields. Proceedings of the International Congress of Mathematicians (Berkeley, 1986), v. 1, 222-244. Amer. Math. Soc. (1987). 4

[52] B. Totaro. Milnor $K$-theory is the simplest part of algebraic $K$-theory. $K$ Theory 6 (1992), 177-189. 19 
[53] B. Totaro. The Chow ring of a classifying space. Algebraic K-theory (Seattle, 1997), 249-281. Proc. Symp. Pure Math. 67, Amer. Math. Soc. (1999). 1, 8, 9, 24, 36, 40,

[54] B. Totaro. Chow groups, Chow cohomology, and linear varieties. Forum of Mathematics, Sigma 2 (2014), e17 (25 pages). 17, 19]

[55] B. Totaro. Group cohomology and algebraic cycles. Cambridge (2014). 1, 2, 8, 9. 38

[56] V. Voevodsky. Triangulated categories of motives over a field. Cycles, transfers, and motivic homology theories, 188-238. Princeton (2000). 13, 14, 15, 17, 18,

[57] V. Voevodsky. Open problems in the motivic stable homotopy theory. I. Motives, polylogarithms and Hodge theory (Irvine, 1998), Part I, 3-34. International Press (2002). 16

[58] V. Voevodsky. Reduced power operations in motivic cohomology. Publ. Math. IHES 98 (2003), 1-57. 36

[59] V. Voevodsky. Motivic Eilenberg-MacLane spaces. Publ. Math. IHES 112 (2010), 1-99. 36

[60] A. Weir. Sylow $p$-subgroups of the classical groups over finite fields with characteristic prime to p. Proc. Amer. Math. Soc. 6 (1955), 529-533. 38

UCLA Mathematics Department, Box 951555, Los Angeles, CA 90095-1555 TOTARO@MATH.UCLA.EDU 